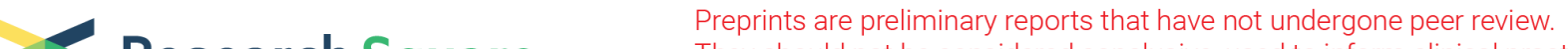 Research Square They should not be considered conclusive, used to inform clinical practice, or referenced by the media as validated information.
}

\section{METTL3 Promotes Lung Adenocarcinoma Tumorigenesis and Inhibits Ferroptosis by Stabilizing SLC7A11 m6A Modification}

\section{Yiming Xu}

Zhejiang University School of Medicine Sir Run Run Shaw Hospital

Dandan Lv

Zhejiang University School of Medicine Sir Run Run Shaw Hospital

Chao Yan

Zhejiang University School of Medicine Sir Run Run Shaw Hospital

Hua Su

Zhejiang University School of Medicine Sir Run Run Shaw Hospital

Xue Zhang

Zhejiang University School of Medicine

\section{Yangfeng Shi}

Zhejiang University School of Medicine Affiliated Hangzhou First People's Hospital

Kejing Ying ( $\boldsymbol{\nabla} 3197061 @ z j u . e d u . c n)$

Zhejiang University School of Medicine Sir Run Run Shaw Hospital https://orcid.org/0000-0001-96423929

\section{Primary research}

Keywords: Lung adenocarcinoma, METTL3, N6-methyladenosine(m6 63 A) modification, ferroptosis, SLC7A11

Posted Date: November 23rd, 2021

DOI: https://doi.org/10.21203/rs.3.rs-1085921/v1

License: (c) (i) This work is licensed under a Creative Commons Attribution 4.0 International License. Read Full License 
Title:

2 METTL3 promotes lung adenocarcinoma tumorigenesis and inhibits

3 ferroptosis by stabilizing SLC7A11 $\mathrm{m}^{6} \mathrm{~A}$ modification

4

5 Yiming $\mathrm{Xu}^{1,2}$, Dandan $\mathrm{Lv}^{1,2}$, Chao Yan ${ }^{1,2}$, Hua Su ${ }^{1,2}$, Xue Zhang ${ }^{3}$, Yangfeng

$6 \quad$ Shi $^{4}$, Kejing Ying ${ }^{1,2}$

71 Department of Respiratory and Critical Medicine, Sir Run Run Shaw Hospital,

8 Zhejiang University School of Medicine, Hangzhou, China

92 Cancer Center, Zhejiang University, Hangzhou, China

103 Department of Pathology and Pathophysiology, Zhejiang University School of

11 Medicine, Hangzhou, China

4 Department of Respiratory and Critical Medicine, Affiliated Hangzhou First People's Hospital, Zhejiang University School of Medicine, Hangzhou, China

\section{Junior corresponding author:}

Yangfeng Shi

Department of Respiratory and Critical Medicine, Affiliated Hangzhou First People's Hospital, Zhejiang University School of Medicine

No.261, Huansha Road, Hangzhou, China.

\section{Email: shiyfzju@163.com}

\section{Senior Corresponding author:}

Kejing Ying

Department of Respiratory and Critical Medicine, Sir Run Run Shaw Hospital, 
Zhejiang University School of Medicine

No.3, Qingchun East Road, Hangzhou, China.

Email: 3197061@zju.edu.cn 7

\section{Abstract}

Background: N6-methyladenosine $\left(m^{6} A\right)$ has emerged as a significant regulator of the progress of various cancers. However, its role in lung adenocarcinoma (LUAD) remains unclear. Here, we explored the biological function and underlying mechanism of methyltransferase-like 3 (METTL3), the main catalyst of $m^{6} A$, in LUAD progression.

Methods: The expression of $\mathrm{m}^{6} \mathrm{~A}, \mathrm{METTL3}, \mathrm{YTHDF} 1$ and SLC7A11 were detected by immunochemistry or/and online datasets in LUAD patients. The effects of METTL3 on LUAD cell proliferation, apoptosis and ferroptosis were assessed through in vitro loss-and gain-of-function experiments. The in vivo effect on tumorigenesis of METTL3 was evaluated using the LUAD cell xenograft mouse model. MeRIP-seq, RNA immunoprecipitation and RNA stability assay were conducted to explore the molecular mechanism of METTL3 in LUAD.

Results: The results showed that the $\mathrm{m}^{6} \mathrm{~A}$ level, as well as the methylase 
METTL3 were both significantly elevated in LUAD patients and lung cancer cells. Functionally, we found that METTL3 could promote proliferation and inhibit ferroptosis in different LUAD cell models, while METTL3 knockdown suppressed LUAD growth in cell-derived xenografts. Mechanistically, solute carrier 7A11 (SLC7A11), the subunit of system $\mathrm{Xc}^{-}$, was identified as the direct target of METTL3 by mRNA-seq and MeRIP-seq. METTL3-mediated $\mathrm{m}^{6} \mathrm{~A}$ modification could stabilize SLC7A11 mRNA and promote its translation, thus promoting LUAD cell proliferation and inhibiting cell ferroptosis, a novel form of programmed cell death. Additionally, we demonstrated that YTHDF1, a $\mathrm{m}^{6} \mathrm{~A}$ reader, was recruited by METTL3 to enhance SLC7A11 $\mathrm{m}^{6} \mathrm{~A}$ modification. Moreover, the expression of YTHDF1 and SLC7A11 were positively correlated with METTL3 and $m^{6} A$ in LUAD tissues.

Conclusions: These findings reinforced the oncogenic role of METTL3 in LUAD progression and revealed its underlying correlation with cancer cell ferroptosis; these findings also indicate that METTL3 is a promising novel target in LUAD diagnosis and therapy.

\section{Key words:}

Lung adenocarcinoma; METTL3; N6-methyladenosine $\left(\mathrm{m}^{6} \mathrm{~A}\right)$ modification; ferroptosis; SLC7A11

\section{Background}


Despite advances in cancer progression and treatment, lung cancer remains occupying the leading cause of cancer-related mortalities worldwide, with an estimated 2.2 million new cases and 1.8 million deaths per year ${ }^{1}{ }^{2}$. Approximately $85 \%$ of patients are diagnosed with non-small-cell lung cancer (NSCLC), of which lung adenocarcinoma (LUAD) is the most frequent histological manifestation ${ }^{3}$. Until now, the overall survival time of LUAD patients remains poor, despite the advancement of surgical resection, chemotherapy, radiotherapy, molecular targeted therapy and immunotherapy. Therefore, further mechanism exploration is necessary for improving the diagnosis and prognosis of LUAD at the outset.

N6-methyladenosine $\left(\mathrm{m}^{6} \mathrm{~A}\right)$, one of the universal modifications of RNA molecules among numerous epigenetic changes, drives multiple biological functions, including tissue development, stemness maintenance and differentiation, DNA damage response and metabolism ${ }^{4-7}$. Alteration of $\mathrm{m}^{6} \mathrm{~A}$ participates in regulating mRNA splicing, export, translation, and stability, which involves three components, including methyltransferases (writers), demethylases (erasers) and RNA-binding proteins (readers) ${ }^{8}$. The $\mathrm{m}^{6} \mathrm{~A}$ methyltransferase complex, contains a core subunit, methyltransferase-like 3 (METTL3), and other accessory regulators including METTL14, Wilms' tumor 1-associating protein (WTAP), VIRMA, RBM15 and ZC3H13, which catalyzes the $\mathrm{m}^{6} \mathrm{~A}$ modification, while demethylases, including fat mass and obesityassociated (FTO) and AlkB homolog 5 (ALKBH5), reverse this effect. 
Additionally, the YT521-B homology (YTH) domain family of proteins (YTHDF1/2/3 and YTHDC1/2), and insulin-like growth factor 2 mRNA binding proteins (IGF2BP1/2/3) are recognized as RNA-binding proteins that bind $\mathrm{m}^{6} \mathrm{~A}$ sites and lead target RNA to different destination ${ }^{9}$. Recently, $\mathrm{m}^{6} \mathrm{~A}$ and its associated proteins were reported to be essentially responsible for tumorigenesis and cancer progression in various cancer types, including lung cancer $^{9-11}$. For example, METTL3 was found essential for TGF- $\beta$-induced epithelial-mesenchymal transition of lung cancer cells ${ }^{12}$; YTHDF2 could promote lung cancer cell growth via facilitating $6 \mathrm{PGD}$ mRNA translation ${ }^{13}$. Yet the biological significance and underlying mechanism of $m^{6} A$ in LUAD remain elusive.

Ferroptosis, the new-found cell death form characterized by iron accumulation and lipid-reactive oxygen species (ROS) within the cell, is distinct both morphologically and functionally from other known forms of cell death including necrosis, apoptosis and autophagy ${ }^{14}$, 15. Increasing evidences have demonstrated that ferroptosis is a crucial regulator of cancer progression and may be harnessed in cancer therapies ${ }^{16,17}$. Liu et al. ${ }^{18}$ found that nuclear factorerythroid 2-like 2 (NRF2) inhibitor (brusatol) could enhance the sensitivity of NSCLC cells to cystine deprivation-induced ferroptosis depending on FOCADFAK signaling, and the combination of brusatol and erastin showed better therapeutic effect of NSCLC. Chen et al. ${ }^{19}$ showed erainin, a novel dibenzyl compound, could induce ferroptotic cell death in lung cancer cells by $\mathrm{Ca}^{2+} / \mathrm{CaM}-$ 
dependent pathway. All of these suggest the potential anti-tumor effect of

112 ferroptosis inducers in LUAD treatment in further researches. Moreover, several

113 studies gradually revealed the potential connections between $\mathrm{m}^{6} \mathrm{~A}$ and

114 ferroptosis. For example, YTHDF1 was found to be closely related to iron

115 metabolism and tumor progression in hypopharyngeal squamous cell

116 carcinoma by targeting TFRC via $\mathrm{m}^{6} \mathrm{~A}$-dependent mechanism ${ }^{20}$. Exosomal

117 miR-4443 could promote cell resistance to cisplatin in NSCLC via FSP1 m A-

118 mediated ferroptosis ${ }^{21}$. Nevertheless, the relationship between $m^{6} A$ and

119 ferroptosis in LUAD still need further exploration.

120 In this study, we found that the level of $m^{6} A$ and the expression of METTL3 were

121 increased in LUAD patients. Then, we demonstrated that the METTL3-

122 mediated $\mathrm{m}^{6} \mathrm{~A}$ modification in LUAD could promote tumorigenesis and inhibit

123 cell ferroptosis via SLC7A11 regulation. Moreover, the METTL3-mediated

124 methylation of SLC7A11 could regulate the stability and translation of SLC7A11

125 mRNA through YTHDF1 recruitment in LUAD cells. We also found that both the

126 expression of YTHDF1 and SLC7A11 were elevated and positively related to

127 METTL3 and $m^{6} A$ levels in LUAD samples, which indicated that METTL3-

128 mediated $\mathrm{m}^{6} \mathrm{~A}$ modification of SLC7A11 illustrated a significant role in human

129 LUAD progression and ferroptosis pathways.

130 2. Materials and Methods

$131 \quad$ 2.1 Patient samples

132 All LUAD tissues and paired adjacent normal lung tissues were obtained from 
LUAD patients who underwent surgery at Sir Run Run Shaw Hospital from January 2019 to January 2020 without previous chemotherapy or radiotherapy.

135 Written informed consent was obtained from each patient in this study, and 136 protocols were approved by the ethical committee of Sir Run Run Shaw

137 Hospital. All specimens were immediately frozen in liquid nitrogen after removal.

138 And all samples were examined by experienced pathologists who confirmed

139 the disease diagnosis.

\subsection{Cell culture and reagents}

NSCLC cell lines (NCl-H1975, A549, PC9, NCl-H322, NCl-H460, SPC-A1, NClH1299), human normal lung epithelial cells (BEAS-2B) were obtained from American Type Culture Collection (ATCC). NSCLC cell lines and BEAS-2B cells were cultured in RPMI-1640 medium (Solarbio, China), supplemented with $10 \%$ fetal bovine serum (FBS), 100 units $/ \mathrm{mL}$ penicillin and $100 \mu \mathrm{g} / \mathrm{mL}$ streptomycin in an incubator with $5 \% \mathrm{CO} 2$ at $37^{\circ} \mathrm{C}$. All human cell lines have been

147 authenticated using short tandem repeat profiling within the last 3 years. All 148 experiments were performed with mycoplasma-free cells.

\section{3 siRNA, shRNA and plasmid constructs}

150 The siRNAs for METTL3 and YTHDF1, and lentivirus for METTL3 knockdown

151 were synthesized by GenePharma (Shanghai, China). The sequences were as

152 follows: siMETTL3\#1 (sense: 5'-GCUACCUGGACGUCAGUAUTT-3', 153 antisense: 5'-AUACUGACGUCCAGGUAGCTT-3'); siMETTL3\#2 (sense: 5'- 
The pEX-3-METTL3 expression plasmid was synthesized by GenePharma

160 (Shanghai, China). The pcDNA3.1-SLC7A11 expression plasmid was synthesized by Genomeditech (Shanghai, China).

\subsection{Cell transfection and lentiviral infection}

For transient transfection, siRNAs and plasmids were transfected into cells using Lipofectamine 3000 (Invitrogen, L3000015) according to the

165 manufacturer's protocol. The medium was refreshed 4-6 hours after 166 transfection. Cells were applied for other assays after 24 to 48 hours' 167 transfection.

For stable transfection, the lentivirus packaged with short hairpin RNA (shRNA) was transduced into cells using polybrene $(5 \mu \mathrm{g} / \mathrm{mL})$. After 48 hours, stably

170 transfected cells were selected with puromycin $(1 \mu \mathrm{g} / \mathrm{mL})$ for $2-7$ days.

\subsection{RNA isolation and real-time quantitative PCR (RT-qPCR)}

172 Total RNA of the indicated cells was extracted according to the manufacturer's

173 protocol using RNA extract reagent (Axygen, AP-MN-MS-RNA-250). The purity

174 and concentration of RNA were determined by measuring the absorbance at 175 A260/280nm using a Nanodrop 2000 (Thermo Fisher Scientific). Reverse transcription of RNA $(1 \mu \mathrm{g})$ was performed prior to cDNA amplification using a 
177 HiFiScript cDNA Synthesis Kit (CWBIO, CW2569). Then, RT-qPCR was 178 performed in a QuantStudio (Applied Biosystem, ABI) system using SYBR 179 Premix Ex Taq ${ }^{\text {TM }}$ II (Takara, RR820A) with primers. $\beta$-actin served as an 180 endogenous control to quantify the relative expression of targeted genes using 181 the $2^{-\Delta \Delta C t}$ method. The primers synthesized by TSINGKE were listed in 182 Supplementary Table S1.

\subsection{Western blot and antibodies}

184 Total protein was extracted using RIPA lysis buffer and was quantified using a 185 BCA protein assay kit (Beyotime, China). Equal amounts of proteins were separated by $10 \%$ sodium dodecyl sulfate-polyacrylamide gel electrophoresis

187 (SDS-PAGE) and then transferred onto polyvinylidene fluoride (PVDF) 188 membranes (Bio-Rad, USA). The membranes were blocked with $5 \%$ nonfat 189 milk for 1 hour at room temperature and then incubated with primary antibodies 190 at $4^{\circ} \mathrm{C}$ overnight. After three washes the following day, the membranes were 191 incubated with HRP-conjugated secondary antibodies (CST, \#7076, \#7074, 192 1:4000 dilution) for 2 hours at room temperature. An ECL detection system 193 (FDbio) was used for visualization. $\beta$-actin or $\alpha$-tubulin served as internal 194 controls. The primary antibodies used in this study were as follows: $\beta$-actin (CST, 195 \#8457, 1:1000), a-tubulin (Sigma, T6199, 1:5000), METTL3 (Abcam, ab195352, 196 1:1000), SLC7A11 (CST, \#12691, 1:1000), and YTHDF1 (Proteintech, 17479197 1-AP, 1:1000). 
Tissues were fixed in $4 \%$ paraformaldehyde and embedded in paraffin. All slides containing tissue sections were incubated with the indicated primary antibodies (METTL3, Abcam, ab195352, 1:500; Ki67, Abcam, ab92742, 1:1000; YTHDF1, Proteintech, 17479-1-AP, 1:200; SLC7A11, Proteintech, 26864-1AP, 1:200) in a humidified chamber at $4^{\circ} \mathrm{C}$ overnight. The Immunohistochemical staining was visualized with diaminobenzidine, with a hematoxylin counterstain to observe nuclei. Protein expression was assessed according to the intensity $(1,0 \%-25 \% ; 2,26 \%-50 \% ; 3,51 \%-75 \% ; 4,76 \%-100 \%)$ and extent of staining ( 0 , negative; 1 , weak; 2 , moderate; 3 , strong) under microscopy. IHC scores were obtained by multiplying the intensity by the extent of staining, and the scores of 0-6 and of 8-12 were classified as low and high expression respectively.

The TUNEL assay was performed according to the instruction of The One Step TUNEL Apoptosis Assay Kit (Beyotime, C1089), and cell nuclei were co-stained with Hoechest 33342.

\subsection{Cell proliferation assays}

For the CCK-8 assay, cells were seeded in 96-well plates at a density of 5000

216 cells per well one day before transfection or ferrostatin-1 (Fer-1, $1 \mu \mathrm{M})$

217 treatment. Then, each well was administered with CCK-8 solution from a kit 218 (APExBIO, USA, K1018) at the indicated time points $(0,1,2,3$ days after treatment). The absorbance at $450 \mathrm{~nm}$ was then measured.

220 The EdU assay was performed using the BeyoClick ${ }^{\mathrm{TM}}$ EdU-555 cell proliferation 
kit (Beyotime, C0075S) according to the manufacturer's instructions. Cells were seeded in 24-well plates at a density of $5 \times 10^{4}$ cells per well one day before

223 treatment.

\subsection{Cell cycle and apoptosis assays}

Cell cycle and apoptosis assays were analyzed by flow cytometry analysis using FACS (BD Biosciences). For the cell cycle analysis, cells were harvested by trypsinization and then fixed in ice-cold $75 \%$ ethanol at $-20^{\circ} \mathrm{C}$ overnight. The next day, the cells were stained with propidium iodide $(\mathrm{PI})$ according to the Cell Cycle Staining Kit (MultiSciences, China, CCS012). Cell cycle distributions were determined by ModFitLT Software. For the cell apoptosis assay, cells were carefully harvested by trypsinization and stained using an Annexin V-FITC/PI Cell Apoptosis Kit (Beyotime, C1062). The percentage of apoptotic cells was analyzed by FlowJo software.

\subsection{Intracellular ROS and malondialdehyde (MDA) measurement}

235 Intracellular ROS levels were detected by the peroxide-sensitive fluorescent probe 2', 7'-dichlorofluorescein diacetate (DCFH-DA, Sigma-Aldrich, D6883). Briefly, after the indicated treatment, cells in 6-well plates were washed with PBS and incubated with $10 \mu \mathrm{M}$ DCFH-DA for 30 minutes under standard

239 conditions. Cells were then washed and collected, and the mean fluorescence intensity of DCFH-DA, which was representative of ROS level, was measured by flow cytometry. The results were analyzed using FlowJo software.

242 For intracellular MDA measurement, lipid Peroxidation MDA assay kit 
(Beyotime, S0131S) was used according to the manufacturer's instructions.

244 The supernatants reacted with thiobarbituric acid (TBA) in each sample, and

245 the levels of MDA were finally evaluated by measuring the absorbance at 532

$246 \mathrm{~nm}$. Then, the MDA levels were normalized to the cell protein contents as

$247 \mathrm{nmol} / \mathrm{mg}$ protein.

\section{$248 \quad 2.11$ Quantification of total m $^{6}$ A RNA}

249 The $\mathrm{m}^{6} \mathrm{~A}$ content of $200 \mathrm{ng}$ RNA extracted from the indicated cells was analyzed 250 using the EpiQuik $\mathrm{m}^{6} \mathrm{~A}$ RNA Methylation Quantification Kit (Colorimetric) 251 (Epigentek, USA, P-9005-48) following the manufacturer's instructions. The $252 \mathrm{~m}^{6}$ A level was quantified by measuring the absorbance of each well at $450 \mathrm{~nm}$, 253 and the standard curve was then used to calculate the $\mathrm{m}^{6} \mathrm{~A}$ level.

\subsection{Methylated RNA immune-precipitation (MeRIP)-seq}

As previously reported 20,22 , total RNA was extracted using TRIzol reagent

256 (Ambion, USA, 223408). The total RNA quality and quantity were analysis of 257 Bioanalyzer 2100 and RNA 6000 Nano LabChip Kit (Agilent, CA, USA) with RIN number $>7.0$. Approximately $50 \mu \mathrm{g}$ of total RNA was subjected to isolation of poly (A) mRNA with poly-T oligo-attached magnetic beads (Invitrogen). The cleaved RNA fragments were incubated for $2 \mathrm{~h}$ at $4^{\circ} \mathrm{C}$ with an $\mathrm{m}^{6} \mathrm{~A}$-specific 261 antibody (Synaptic Systems, Germany, No. 202003) in IP buffer (50 mM Tris$\mathrm{HCl}, 750 \mathrm{mM} \mathrm{NaCl}$ and $0.5 \%$ Igepal CA-630) supplemented with BSA. The mixture was then incubated with protein-A beads and eluted with elution buffer

$264\left(1 \times\right.$ IP buffer and $\left.6.7 \mathrm{mM} \mathrm{m}^{6} \mathrm{~A}\right)$. Eluted RNA was precipitated by $75 \%$ ethanol. 
Eluted $\mathrm{m}^{6} \mathrm{~A}$-containing fragments (IP) and untreated input control fragments are converted to the final cDNA library in accordance with a strand-specific library preparation by the dUTP method. Finally, we performed $2 \times 150$ bp paired-end sequencing on an Illumina NovaSeq ${ }^{\text {TM }} 6000$ platform at LC-BIO Bio-tech Itd (Hangzhou, China) according to the vendor's recommended protocol.

\subsection{RNA immune-precipitation (RIP)}

271 The RIP assay was performed using a Magna RIPTM RNA-Binding Protein 272 Immunoprecipitation Kit (Millipore, USA, 17-700) according to the 273 manufacturer's instructions. Briefly, the indicated cell lysates were collected and 274 incubated with magnetic bead protein A/G (CS203178) coated with $5 \mu \mathrm{g}$ of 275 control IgG antibody (PP64B), anti-m6 A antibody (Synaptic Systems, 202003), 276 or anti-YTHDF1 antibody (Proteintech, 17479-1-AP) with rotation at $4^{\circ} \mathrm{C}$ 277 overnight. The next day, RNA was purified and extracted using the phenol: 278 chloroform: isoamyl alcohol method. The relative expression of SLC7A11 was 279 detected by RT-qPCR. IP enrichment was normalized to the input yielded from 280 the same number of cells.

\subsection{RNA stability assay}

282 RNA stability assays were performed as previously described ${ }^{23}$. Briefly, cells 283 were treated with actinomycin D (APExBIO, A4448) for $0 \mathrm{~h}, 3 \mathrm{~h}$, or $6 \mathrm{~h}$ at a final concentration of $5 \mu \mathrm{g} / \mathrm{mL}$. Then, total RNA was extracted for RT-qPCR to quantify the relative expression of SLC7A11 mRNA. The degradation rate of mRNA ( $\left.K_{\text {decay }}\right)$ was calculated using the following equation: $\ln \left(C / C_{0}\right)=-K_{\text {decayt }}$. 
The half-life $\left(t_{1 / 2}\right)$ of mRNA was calculated using the equation: $\ln (1 / 2)=$ -

$288 K_{\text {decayt }} t_{1 / 2}$

\subsection{Animal experiment}

290 Female BALB/c-nude mice (5 weeks of age) were used for xenografts models 291 and raised under specific pathogen-free conditions. The animal experiments were approved by The Institutional Animal Care and Use Committee of Zhejiang

293 University. For the subcutaneous xenograft model, PC9 cells stably transfected with METTL3 knockdown (shMETTL3) or negative control (shNC) shRNA $295\left(5 \times 10^{6}\right.$ cells per mouse, $\left.n=6\right)$ were suspended in $200 \mu$ PBS with $50 \%$ Matrigel matrix (Corning, USA, 354234) and then injected into one side of the axilla of

297 nude mice. Tumor growth and volume were measured every 3 days, and the 298 tumor volume was assessed using the formula: volume $\left(\mathrm{mm}^{3}\right)=$ longer 299 diameter $x$ shorter diameter ${ }^{2} / 2$. After 3 weeks, the mice were sacrificed, and 300 tumor weight was determined.

\subsection{Statistical analysis}

302 Data between two groups were analyzed using two-tailed unpaired Student's ttest while one-way or two-way ANOVA was used for multiple comparisons using GraphPad Prism 8.0 and SPSS 20.0. The data are showed as the mean \pm

305 standard deviation (SD) from at least three independent experiments. A P-value 306 less than 0.05 was considered statistically significant. 
310 To investigate the role of $m^{6} A$ modification in LUAD, we first evaluated the $m^{6} A$

311 level in LUAD tissues by immunohistochemistry (IHC). The $\mathrm{m}^{6} \mathrm{~A}$ level was

312 increased compared with adjacent normal tissues (Figure $1 \mathrm{~A}$ ). Since $\mathrm{m}^{6} \mathrm{~A}$

313 writers are the main catalysts that lead $\mathrm{m}^{6} \mathrm{~A}$ modifications ${ }^{9}$, we analyzed

314 different writers, including METTL3, METTL14, WTAP, ZC3H13 and RBM15, in

315 The Cancer Genome Atlas (TCGA) database using the UALCAN platform ${ }^{24}$.

316 METTL3 was the most significantly elevated gene in LUAD samples (Figure 1B,

317 Figure S1A-D). In addition, the elevated expression of METTL3 was also

318 confirmed in the Gene Expression Omnibus (GEO) database (GSE2514)

319 (Figure 1C) and our LUAD tissues by IHC (Figure 1D). In the NSCLC cell lines,

320 RT-qPCR and western blot also indicated that both the mRNA and protein levels

321 of METTL3 were elevated compared with those in BEAS-2B cells, which are

322 normal human lung epithelial cells (Figure 1E, F). Consistently, the mRNA

323 content of $\mathrm{m}^{6} \mathrm{~A}$ in LUAD cells was much higher than that in BEAS-2B cells, as

324 measured by $\mathrm{m}^{6} \mathrm{~A}$ quantitative measurement (Figure 1G). Furthermore, 325 knockdown of METTL3 noticeably decreased the $\mathrm{m}^{6} \mathrm{~A}$ level in LUAD cells, while

326 METTL3 overexpression had the opposite effect (Figure $1 \mathrm{H}, \mathrm{I})$. The transfection

327 efficiencies were confirmed by western blot (Figure 1J, K). These results 328 collectively reveal that METTL3 regulate the $\mathrm{m}^{6} \mathrm{~A}$ level in LUAD patients and 329 NSCLC cells. 
332 To explore the correlation of METTL3 level and the malignancy of LUAD, we 333 also knocked down METTL3 in PC9 and H1975 cells using two specific small 334 interferon RNAs (siRNAs, named si\#1 and si\#2). The efficiency of knockdown 335 was confirmed by western blot (Figure 2A). LUAD cell growth decreased remarkably upon METTL3 knockdown, as determined by CCK-8 assays, while 337 overexpression increased cell growth (Figure 2B, C). Furthermore, EdU 338 staining demonstrated that METTL3 knockdown inhibited cell proliferation, in 339 contrast, overexpression substantially promoted cell proliferation (Figure 2D, E). 340 Since METTL3 distinctly regulated LUAD cell proliferation, we then assessed 341 its impact on the cell cycle. As substantiated by flow cytometry analysis, the cell 342 cycle was arrested in G0/G1 phase and the number of cells in S/G2 phase was 343 reduced due to METTL3 knockdown (Figure 2F); consistently, METTL3 344 overexpression resulted in the opposite effect (Figure 2G). In addition, the 345 proportion of apoptotic cells was increased when METTL3 was knocked down, 346 while overexpression decreased it (Figure $2 \mathrm{H}, \mathrm{I}$ ). To further evaluate the 347 oncogenic role of METTL3 in LUAD in vivo, we applied subcutaneous xenograft 348 models established with stable METTL3 knockdown (shMETTL3) PC9 cells 349 and control (shNC) PC9 cells. Consistent with the in vitro results, the tumors of 350 the METTL3-deficient group grew more slowly than those of the control group 351 (Figure 2J, K). Moreover, the average tumor volume and tumor weight at 352 sacrifice were both remarkably decreased in the shMETTL3 group compared 
with the shNC group (Figure 2L). Then, we evaluated the cell proliferation marker Ki-67 by IHC and the proportion of apoptotic cells by TUNEL assay in

355 these solid tumors. As expected, the IHC score for Ki-67 in shMETTL3 group was significantly reduced while the proportion of apoptotic cells was increased compared with those in the control group (Figure $2 \mathrm{M}, \mathrm{N}$ ). Overall, these lossand gain-of-function assays confirm that METTL3 promotes LUAD proliferation and inhibits apoptosis both in vitro and in vivo.

3.3SLC7A11 is a target of METTL3 in LUAD depending on its $\mathrm{m}^{6} \mathrm{~A}$ methyltransferase activity

To determine whether the mechanism of the oncogenic role of METTL3 in LUAD depends on its $m^{6} A$ methyltransferase activity, RNA sequencing (RNAseq) and $\mathrm{m}^{6} \mathrm{~A}$-modified RNA immunoprecipitation sequencing (MeRIP-seq) were performed in PC9 cells with stable METTL3 knockdown and control PC9 cells. GO analysis of MeRIP-seq revealed that METTL3 and METTL3-mediated $\mathrm{m}^{6} \mathrm{~A}$ methylation participate in multiple biological processes and molecular functions as well as cellular components (Figure 3A). Then, we further screened genes with hypomethylated $\mathrm{m}^{6} \mathrm{~A}$ with decreased expression in METTL3 knockdown cells $(p<0.05)$ and filtered them according to downregulated genes

371 in the RNA-seq analysis $(p<0.05)$. There were 27 genes chosen, and in our verification qPCR analysis, SLC7A11 (also known as xCT) was found to be the only gene that down expressed consistently (fold change <0.5) in H1975 and

374 PC9 cells when METTL3 was knocked down (Figure 3B, C). Besides, the $\mathrm{m}^{6} \mathrm{~A}$ 
peaks of SLC7A11 were remarkably reduced in METTL3 knockdown PC9 cells compared with control cells (Figure 3D). Thus, SLC7A11 was selected as a further candidate target of METTL3 in LUAD. In our validation assays, the protein levels of SLC7A11 were also found significantly decreased after METTL3 knockdown in H1975 and PC9 cells (Figure 3E). Conversely, overexpression of METTL3 in H322 cells indeed showed the opposite effect (Figure 3F, G). Moreover, we measured SLC7A11 level by IHC in xenograft tumors, and found that the expression level of SLC7A11 in shMETTL3 group was significantly reduced compared with control group (Figure H). Consistent with MeRIP-seq, MeRIP-qPCR analysis data showed that $\mathrm{m}^{6}$ A SLC7A11 RNA was decreased upon METTL3 knockdown in both H1975 and PC9 cells, while overexpression substantially increased this (Figure $3 \mathrm{l}, \mathrm{J}$ ), which indicated that the $\mathrm{m}^{6} \mathrm{~A}$ modification of SLC7A11 directly affected its expression. To investigate whether the $\mathrm{m}^{6} \mathrm{~A}$ modification affects the stability of SLC7A11 mRNA, we next performed RNA decay assays using the transcription inhibitor actinomycin $D$ in different groups of cells at the indicated times. As the curves showed, METTL3 knockdown highly accelerated the half-life of SLC7A11 mRNA decay, while overexpression showed the opposite effect (Figure 3K, L), which suggested its impact on SLC7A11 stability. In all, these results support the finding that SLC7A11 acts as a target of METTL3 in LUAD cells, which is dependent on its $\mathrm{m}^{6} \mathrm{~A}$ methyltransferase activity.

\subsection{METTL3-mediated $\mathrm{m}^{6} \mathrm{~A}$ modification of SLC7A11 promotes LUAD}


SLC7A11, a subunit of system $\mathrm{Xc}^{-}$, was recently shown to be overexpressed in multiple human cancers and to promote tumor progression partly by suppressing ferroptosis ${ }^{25}$. Intriguingly, the KEGG pathway enrichment of MeRIP-seq also revealed that ferroptosis was a significant pathway (Figure 4A). Considering the prominence of ferroptosis among these pathways enriched by 403 this MeRIP-seq, as well as the role of SLC7A11 in ferroptosis, we tried to explore the METTL3 regulation of SLC7A11 in ferroptosis of LUAD. ROS production and lipid peroxidation are critical mechanisms of ferroptosis-induced cell death ${ }^{16}$. Therefore, we measured intracellular ROS levels using DFCH-DA and the oxidative stress marker malondialdehyde (MDA). As expected, ROS accumulation and lipid peroxidation were much higher in METTL3 knockdown LUAD cells; likewise, METTL3 overexpression in H322 cells exhibited the opposite phenomenon (Figure 4B-E). Furthermore, the elevated level in METTL3 stable knockdown cells could be completely attenuated by the presence of ferroptosis inhibitor ferrostatin-1 (Fer-1), or by SLC7A11 overexpression (Figure 4F-H). The efficiency of SLC7A11 overexpression was confirmed by western blot (Figure 4I). Moreover, CCK-8 assays showed that METTL3 knockdown could significantly inhibit H1975 and PC9 cell proliferation; however, this effect could be largely reversed by treatment with Fer-1 or of SLC7A11 overexpression (Figure 4J). This was also verified by EdU assays in 418 PC9 cells (Figure 4K). Likewise, the promotion effect of cell apoptosis caused 
by METTL3 knockdown in PC9 cells was reduced with Fer-1 treatment or

SLC7A11 overexpression as well (Figure 4L). Taken together, these findings

421 strongly demonstrate that METTL3 can inhibit LUAD cell ferroptosis via

422 SLC7A11 $\mathrm{m}^{6} \mathrm{~A}$ modification.

3.5 METTL3 recruits YTHDF1 to regulate SLC7A11 mRNA stability and

\section{translation}

425 The above results show that METTL3 regulates SLC7A11 expression to 426 promote LUAD, yet the modification mechanism remains to be elucidated. 427 YTHDF1 is recognized as one of the most important $m^{6} A$ readers responsible 428 for mRNA translation ${ }^{26}$. Recent studies inspired us that METTL3 could recruit 429 YTHDF1 to promote their target transcript stability ${ }^{27,28}$. Thus, we knocked down 430 YTHDF1 in PC9 cells by siRNA transfection, and the knockdown efficiency was 431 validated by RT-qPCR and western blot (Figure 5A, B). The downregulation of 432 YTHDF1 markedly reduced the mRNA and protein expression of SLC7A11 in 433 PC9 cells (Figure 5C, D). Moreover, the half-life of SLC7A11 mRNA was also 434 reduced in YTHDF1-downregulated PC9 cells (Figure 5E), which indicated the 435 essential role of YTHDF1 in regulating SLC7A11 mRNA stability. Furthermore, 436 RIP-qPCR analysis showed that YTHDF1 bound directly to SLC7A11 mRNA, 437 while METTL3 knockdown significantly reduced this binding efficiency (Figure $4385 F)$. In addition, we found that the downregulated protein levels of SLC7A11 in 439 YTHDF1-knockdown H322 cells could not be rescued by METTL3 overexpression (Figure 5G), which implied that YTHDF1 was requisite for 
METTL3-mediated SLC7A11 $\mathrm{m}^{6} \mathrm{~A}$ modification. Moreover, the ROS levels and

442 the proportion of apoptosis cells were significantly increased when YTHDF1

443 was knocked down in H322 cells, while METTL3 overexpression had no

444 discernible effect on these. (Figure $5 \mathrm{H}, \mathrm{I}$ ). In conclusion, our data reveal that

445 YTHDF1 increase SLC7A11 expression through recognizing METTL3-

446 mediated $\mathrm{m}^{6} \mathrm{~A}$-methylated SLC7A11 mRNA and enhancing its stability and

447 translation.

3.6 METTL3-mediated $\mathrm{m}^{6} \mathrm{~A}$ modification of SLC7A11 is clinically related to

\section{LUAD progression}

450 To confirm the clinical significance of METTL3/SLC7A11 axis in LUAD, we next 451 investigated the expression of YTHDF1 and SLC7A11 in LUAD human samples. 452 As expected, both the expression of YTHDF1 and SLC7A11 were observably 453 elevated in our LUAD samples by IHC and in the TCGA database using the 454 UALCAN platform (Figure 6A-D). What's more, the expression of METTL3 and $455 \mathrm{~m}^{6} \mathrm{~A}$ were positively correlated with the expression of YTHDF1 and SLC7A11 in our LUAD clinical samples respectively (Figure 6E, F). Moreover, LUAD 457 patients with higher levels of SLC7A11 had poorer OS using the online analysis 458 tool Kaplan-Meier Plotter (http://kmplot.com/analysis/) (Figure 6G). Therefore, 459 these data suggest that the METTL3-mediated $\mathrm{m}^{6} \mathrm{~A}$ modification of SLC7A11 460 promotes human LUAD progression significantly.

\section{Discussion}

462 In lung cancer, epigenetic alterations, such as DNA methylation, noncoding 
RNA expression, chromatin remodeling and posttranscriptional regulators, are key components in each step of tumor pathogenesis ${ }^{29,}{ }^{30}$. Studies have showed that resistance to ferroptosis, the iron-dependent, nonapoptotic form of programmed cell death, is observed in many cell types and is closely related to the pathophysiological processes of many diseases, including neurodegeneration ${ }^{31}$, liver fibrosis ${ }^{32}$, ischemia/reperfusion-related damage ${ }^{33-35}$ and various human cancers, as well as lung cancer ${ }^{17}$. During the past few years, accumulating evidence has revealed the essential role of the $\mathrm{m}^{6} \mathrm{~A}$ modification in human cancers ${ }^{9}, 11$. Abnormal levels of $\mathrm{m}^{6} \mathrm{~A}$ as well as its related proteins, including writers, erasers and readers, exhibit a strong correlation with tumor pathogenesis and progression. However, the $\mathrm{m}^{6} \mathrm{~A}$ modification in LUAD still remains unclear. Recently, there have been several findings showing that $\mathrm{m}^{6} \mathrm{~A}$ was closely linked with ferroptosis in cancers as well, which unveiled the great value of exploring the potential role of $\mathrm{m}^{6} \mathrm{~A}$ in LUAD ferroptosis.

Our study showed elevated $\mathrm{m}^{6} \mathrm{~A}$ levels in LUAD patients and cells, and METTL3 was the most significantly increased writer in LUAD compared with METTL14, WTAP, ZC3H13 or RBM15 in LUAD. Recent evidence has found that METTL3, depending on its methyltransferase activity, serves as an oncogene or tumor suppressor in different cancers ${ }^{36}$. For example, the depletion of METTL3 in acute myeloid leukemia (AML) cells induced cell differentiation and apoptosis through METTL3-mediated $\mathrm{m}^{6} \mathrm{~A}$ modification on MYC, BCL2 and PTEN mRNA, thus delaying AML progression ${ }^{37}$. In colorectal cancer, METTL3 expression was 
found to be much higher in patients with higher FDG uptake, promoting cancer progression, which depends on cell glycolytic metabolism, by stabilizing HK2 and GLUT1 expression in an $\mathrm{m}^{6} \mathrm{~A}-$ IGF2BP2/3-dependent manner ${ }^{23}$. In contrast, Jia et al. reported that ocular melanoma showed decreased $\mathrm{m}^{6} \mathrm{~A}$ levels due to downregulation of METTL3 and demonstrated that METTL3-mediated $\mathrm{m}^{6} \mathrm{~A}$ modification could promote the translation of tumor suppressor gene HINT2 ${ }^{38}$. Here, corresponded with previous researches in lung cancer ${ }^{12,39}$, our study demonstrated that METTL3 played an oncogenic role in LUAD tumorigenesis. Firstly, we conducted a series of loss-and gain-of-function assays in LUAD cells investigating the biological impact of METTL3. Results showed that METTL3 knockdown in H1975 and PC9 cells promoted cell proliferation and inhibits apoptosis, while overexpression in $\mathrm{H} 322$ cells exerted the opposite effect. Subsequently, METTL3 suppression in cell-derived xenografts exhibited a significant inhibitory effect in tumor growth, which further indicated the oncogenic role of METTL3 in LUAD tumorigenesis.

To further clarify the molecular mechanism of METTL3 in LUAD, we performed RNA-seq and MeRIP-seq analysis with stable METTL3 knockdown cells. Intriguingly, the KEGG analysis showed that ferroptosis was the closely correlated pathway. Meanwhile, SLC7A11, one reported regulator of ferroptosis was screened as the significant differently expressed gene affected by the level of METTL3. Our subsequent validation assays confirmed that METTL3 upregulated SLC7A11 mRNA methylation and enhanced its stability and 
translation, which was consistent with a previous study ${ }^{40}$. As is previously shown, SLC7A11 overexpression in cancer cells promotes ferroptosis resistance and thus influencing cancer growth, invasion, and metastasis and led to an unfavorable prognosis ${ }^{41}$. Additionally, SLC7A11 was also found to be essential for tumor growth by relieving oxidative stress in some oncogenic KRAS-mutant cancers, including pancreatic ductal adenocarcinoma, colorectal adenocarcinoma and LUAD ${ }^{42,43}$.

514 Since iron-dependent ROS production and the accumulation of lipid peroxidation products are the main characteristics of ferroptosis ${ }^{14}$, 44 , we assessed intracellular ROS and lipid peroxidation levels to further explore the

517 potential relation among METTL3, SLC7A11 and ferroptosis. We found that METTL3 knockdown significantly promoted LUAD cell ferroptosis, but further treated with ferroptosis inhibitor, ferrostatin-1, which could inversely inhibits lipid preoxidation $^{45}$, almost reversed the increase of LUAD cell ferroptosis and the

521 decrease of LUAD cell proliferation caused by METTL3 deficiency. Additionally, we also demonstrated that SLC7A11 overexpression could partly rescue these effects in METTL3 knockdown LUAD cells, which represented that SLC7A11 acted as a target of METTL3 in LUAD in terms of both function and mechanism.

525 Recent studies have illustrated that $m^{6} A$ readers can recognize the $m^{6} A$ sites of mRNA transcripts and participate in multiple processes of RNA metabolism ${ }^{46}$.

527 Among these, YTHDF1 tends to stabilize the transcript and promote mRNA 528 translation, while several studies have demonstrated that METTL3 enhances 
targeted mRNA stability and translation in a YTHDF1-dependent manner in

530 cervical cancer ${ }^{27}$, oral squamous cell carcinoma ${ }^{28}$ and liver cancer ${ }^{47}$. Besides,

531 YTHDF1 was also found to be an oncogene in NSCLC, as it regulated the

532 translational efficiency of CDK2, CDK4, and cyclin D122. As expected, our 533 validation experiments confirmed that METTL3 promoted SLC7A11 mRNA 534 stability and translation through YTHDF1 recruitment in LUAD cells. Moreover, 535 the expression of YTHDF1 and SLC7A11 in LUAD tissues were positively 536 correlated with METTL3 and $\mathrm{m}^{6} \mathrm{~A}$ level, which indicated the clinical significance 537 of METTL3-mediated $\mathrm{m}^{6} \mathrm{~A}$ modification of SLC7A11 in LUAD progression.

\section{Conclusion}

539 In summary, our work supports the oncogenic role of METTL3 in LUAD

540 tumorigenesis and reveals its regulatory role in ferroptosis. Mechanistically, 541 METTL3 promotes LUAD progression through SLC7A11 $\mathrm{m}^{6} \mathrm{~A}$ modification in a

542 YTHDF1-dependent manner. Moreover, SLC7A11 expression is correlated with 543 poor prognosis of LUAD patients. Thus, targeting METTL3 and METTL3544 mediated $\mathrm{m}^{6} \mathrm{~A}$ modification of SLC7A11 might be promising diagnostic and 545 therapeutic strategy for LUAD.

\section{Abbreviations:}

548 Fer-1, ferrostatin-1; IHC, immunochemistry; LUAD, lung adenocarcinoma; m6A, 549 N6-methyladenosine; MDA, malondialdehyde; MeRIP, methylated RNA 550 immune-precipitation; METTL3, methyltransferase-like 3; NSCLC, non-small- 
cell lung cancer; RIP, RNA immune-precipitation; ROS, reactive oxygen species;

552 RT-qPCR, real-time quantitative PCR; SLC7A11, solute carrier 7A11; TCGA,

553 The Cancer Genome Atlas; YTHDF, YT521-B homology domain family of

554 proteins

555

556 Declarations

557 Ethics approval and consent to participate

558 The study protocols were approved by the ethical committee of Sir Run Run

559 Shaw Hospital. Written informed consent was obtained from each patient in this

560 study. The protocols for animal studies were approved by The Institutional

561 Animal Care and Use Committee of Zhejiang University.

562 Consent for publication

563 Not applicable.

564 Availability of data and materials

565 The data that support the findings of this study are available on request from

566 the corresponding author.

567 Competing interests

568 The authors declare that they have no competing interests.

\section{Funding}

570 This study was supported by National Natural Science Foundation of China

571 (81970049, 82170054), Natural Science Foundation of Zhejiang Province

572 (Y21H010014). 


\section{Authors' contribution}

$\mathrm{YX}, \mathrm{YS}$ and $\mathrm{KY}$ conceived and designed the study. $\mathrm{YX}, \mathrm{DL}, \mathrm{CY}$ and $\mathrm{HS}$ performed experiments, analyzed the data and prepared figures. YX and DL drafted the manuscript. $X Z, Y S$ and $K Y$ revised the final manuscript. All authors read and approved the final manuscript.

\section{Acknowledgements}

We thank American Journal Experts for help in English grammar and fluency.

\section{References}

1. Sung H, Ferlay J, Siegel R L, Laversanne M, Soerjomataram I, Jemal A, Bray F. Global Cancer Statistics 2020: GLOBOCAN Estimates of Incidence and Mortality Worldwide for 36 Cancers in 185 Countries [J]. CA: A Cancer Journal for Clinicians, 2021, 71(3): 209-249.

2. Ferlay J, Colombet M, Soerjomataram I, Parkin D M, Piñeros M, Znaor A, Bray F. Cancer statistics for the year 2020: An overview [J]. International Journal of Cancer, 2021, 149(4): 778-789.

3. Herbst R S, Morgensztern D, Boshoff $C$. The biology and management of non-small cell lung cancer [J]. Nature, 2018, 553(7689): 446-454.

4. Dominissini D, Moshitch-Moshkovitz S, Schwartz S, Salmon-Divon M, Ungar L, Osenberg S, Cesarkas K, Jacob-Hirsch J, Amariglio N, Kupiec M, Sorek R, Rechavi G. Topology of the human and mouse m6A RNA methylomes revealed by m6A-seq [J]. Nature, 2012, 485(7397): 201-206. 
595

596

597

598

599

600

601

602

603

604

605

606

607

608

609

610

611

612

613

614

615

616

5. Frye M, Harada B T, Behm M, He C. RNA modifications modulate gene expression during development [J]. Science, 2018, 361(6409): 1346-1349.

6. Wang Y, Li Y, Toth J I, Petroski M D, Zhang Z, Zhao J C. N6methyladenosine modification destabilizes developmental regulators in embryonic stem cells [J]. Nat Cell Biol, 2014, 16(2): 191-198.

7. Zhang H, Shi X, Huang T, Zhao X, Chen W, Gu N, Zhang R. Dynamic landscape and evolution of m6A methylation in human [J]. Nucleic Acids Res, 2020, 48(11): 6251-6264.

8. Roundtree I A, Evans M E, Pan T, He C. Dynamic RNA Modifications in Gene Expression Regulation [J]. Cell, 2017, 169(7): 1187-1200.

9. Huang $\mathrm{H}$, Weng $\mathrm{H}$, Chen J. m(6)A Modification in Coding and Non-coding RNAs: Roles and Therapeutic Implications in Cancer [J]. Cancer Cell, 2020, 37(3): 270-288.

10. Deng X, Su R, Weng H, Huang H, Li Z, Chen J. RNAN(6)-methyladenosine modification in cancers: current status and perspectives [J]. Cell research, 2018, 28(5): 507-517.

11. Wang T, Kong S, Tao M, Ju S. The potential role of RNA N6methyladenosine in Cancer progression [J]. Mol Cancer, 2020, 19(1): 88-88.

12. Wanna-udom S, Terashima M, Lyu H, Ishimura A, Takino T, Sakari M, Tsukahara T, Suzuki T. The m6A methyltransferase METTL3 contributes to Transforming Growth Factor-beta-induced epithelial-mesenchymal transition of lung cancer cells through the regulation of JUNB [J]. Biochemical and 
Biophysical Research Communications, 2020, 524(1): 150-155.

13. Sheng H, Li Z, Su S, Sun W, Zhang X, Li L, Li J, Liu S, Lu B, Zhang S, Shan C. YTH domain family 2 promotes lung cancer cell growth by facilitating 6phosphogluconate dehydrogenase mRNA translation [J]. Carcinogenesis, 2020, 41(5): 541-550.

14. Dixon S J, Lemberg K M, Lamprecht M R, Skouta R, Zaitsev E M, Gleason C E, Patel D N, Bauer A J, Cantley A M, Yang W S, Morrison B, 3rd, Stockwell B R. Ferroptosis: an iron-dependent form of nonapoptotic cell death [J]. Cell, 2012, 149(5): 1060-1072.

15. Li J, Cao F, Yin H-L, Huang Z-J, Lin Z-T, Mao N, Sun B, Wang G. Ferroptosis: past, present and future [J]. Cell Death Dis, 2020, 11(2): 88-88.

16. Liang C, Zhang X, Yang M, Dong X. Recent Progress in Ferroptosis Inducers for Cancer Therapy [J]. Advanced Materials, 2019, 31(51): 1904197. 17. Mou Y, Wang J, Wu J, He D, Zhang C, Duan C, Li B. Ferroptosis, a new form of cell death: opportunities and challenges in cancer [J]. J Hematol Oncol, 2019, 12(1): 34-34.

18. Liu P, Wu D, Duan J, Xiao H, Zhou Y, Zhao L, Feng Y. NRF2 regulates the sensitivity of human NSCLC cells to cystine deprivation-induced ferroptosis via FOCAD-FAK signaling pathway [J]. Redox Biol, 2020, 37(101702.

19. Chen P, Wu Q, Feng J, Yan L, Sun Y, Liu S, Xiang Y, Zhang M, Pan T, Chen X, Duan T, Zhai L, Zhai B, Wang W, Zhang R, Chen B, Han X, Li Y, Chen L, Liu Y, Huang X, Jin T, Zhang W, Luo H, Chen X, Li Y, Li Q, Li G, Zhang Q, Zhuo L, 
Yang Z, Tang H, Xie T, Ouyang X, Sui X. Erianin, a novel dibenzyl compound in Dendrobium extract, inhibits lung cancer cell growth and migration via calcium/calmodulin-dependent ferroptosis [J]. Signal Transduct Target Ther, 2020, 5(1): 51 .

20. Ye J, Wang Z, Chen X, Jiang X, Dong Z, Hu S, Li W, Liu Y, Liao B, Han W, Shen J, Xiao M. YTHDF1-enhanced iron metabolism depends on TFRC m(6)A methylation [J]. Theranostics, 2020, 10(26): 12072-12089.

21. Song Z, Jia G, Ma P, Cang S. Exosomal miR-4443 promotes cisplatin resistance in non-small cell lung carcinoma by regulating FSP1 m6A modification-mediated ferroptosis [J]. Life Sciences, 2021, 276(119399.

22. Jin W, Xu H-X, Zhang S-R, Li H, Wang W-Q, Gao H-L, Wu C-T, Xu J-Z, Qi Z-H, Li S, Ni Q-X, Liu L, Yu X-J. Tumor-Infiltrating NETs Predict Postsurgical Survival in Patients with Pancreatic Ductal Adenocarcinoma [J]. Annals of Surgical Oncology, 2019, 26(2): 635-643.

23. Shen C, Xuan B, Yan T, Ma Y, Xu P, Tian X, Zhang X, Cao Y, Ma D, Zhu X, Zhang Y, Fang J-Y, Chen H, Hong J. m(6)A-dependent glycolysis enhances colorectal cancer progression [J]. Mol Cancer, 2020, 19(1): 72-72.

24. Chandrashekar D S, Bashel B, Balasubramanya S A H, Creighton C J, Ponce-Rodriguez I, Chakravarthi B V S K, Varambally S. UALCAN: A Portal for Facilitating Tumor Subgroup Gene Expression and Survival Analyses [J]. Neoplasia, 2017, 19(8): 649-658.

25. Koppula P, Zhuang L, Gan B. Cystine transporter SLC7A11/xCT in cancer: 
661

662

663

ferroptosis, nutrient dependency, and cancer therapy [J]. Protein \& Cell, 2020, 26. Shi R, Ying S, Li Y, Zhu L, Wang X, Jin H. Linking the YTH domain to cancer: the importance of YTH family proteins in epigenetics [J]. Cell Death Dis, 2021, 12(4): 346-346.

27. Wang Q, Guo X, Li L, Gao Z, Su X, Ji M, Liu J. N(6)-methyladenosine METTL3 promotes cervical cancer tumorigenesis and Warburg effect through YTHDF1/HK2 modification [J]. Cell Death Dis, 2020, 11(10): 911-911.

28. Zhao W, Cui Y, Liu L, Ma X, Qi X, Wang Y, Liu Z, Ma S, Liu J, Wu J. METTL3 Facilitates Oral Squamous Cell Carcinoma Tumorigenesis by Enhancing c-Myc Stability via YTHDF1-Mediated m(6)A Modification [J]. Mol Ther Nucleic Acids, $2020,20(1-12$

29. Duruisseaux M, Esteller M. Lung cancer epigenetics: From knowledge to applications [J]. Seminars in Cancer Biology, 2018, 51(116-128.

30. Bennett R L, Licht J D. Targeting Epigenetics in Cancer [J]. Annu Rev Pharmacol Toxicol, 2018, 58(187-207.

31. Masaldan S, Bush A I, Devos D, Rolland A S, Moreau C. Striking while the iron is hot: Iron metabolism and ferroptosis in neurodegeneration [J]. Free Radical Biology and Medicine, 2019, 133(221-233.

32. Zhang Z, Guo M, Li Y, Shen M, Kong D, Shao J, Ding H, Tan S, Chen A, Zhang F, Zheng S. RNA-binding protein ZFP36/TTP protects against ferroptosis by regulating autophagy signaling pathway in hepatic stellate cells [J]. Autophagy, 2020, 16(8): 1482-1505. 
33. Li Y, Cao Y, Xiao J, Shang J, Tan Q, Ping F, Huang W, Wu F, Zhang H, Zhang X. Inhibitor of apoptosis-stimulating protein of p53 inhibits ferroptosis and alleviates intestinal ischemia/reperfusion-induced acute lung injury [J]. Cell Death \& Differentiation, 2020, 27(9): 2635-2650.

34. Li Y, Feng D, Wang Z, Zhao Y, Sun R, Tian D, Liu D, Zhang F, Ning S, Yao J, Tian X. Ischemia-induced ACSL4 activation contributes to ferroptosismediated tissue injury in intestinal ischemia/reperfusion [J]. Cell Death Differ, 2019, 26(11): 2284-2299.

35. Su L, Jiang X, Yang C, Zhang J, Chen B, Li Y, Yao S, Xie Q, Gomez H, Murugan R, Peng Z. Pannexin 1 mediates ferroptosis that contributes to renal ischemia/reperfusion injury [J]. J Biol Chem, 2019, 294(50): 19395-19404.

36. Zeng C, Huang W, Li Y, Weng H. Roles of METTL3 in cancer: mechanisms and therapeutic targeting [J]. J Hematol Oncol, 2020, 13(1): 117-117.

37. Vu L P, Pickering B F, Cheng Y, Zaccara S, Nguyen D, Minuesa G, Chou T, Chow A, Saletore Y, MacKay M, Schulman J, Famulare C, Patel M, Klimek V M, Garrett-Bakelman F E, Melnick A, Carroll M, Mason C E, Jaffrey S R, Kharas M G. The N(6)-methyladenosine (m(6)A)-forming enzyme METTL3 controls myeloid differentiation of normal hematopoietic and leukemia cells [J]. Nat Med, 2017, 23(11): 1369-1376.

38. Jia R, Chai P, Wang S, Sun B, Xu Y, Yang Y, Ge S, Jia R, Yang Y-G, Fan X. m(6)A modification suppresses ocular melanoma through modulating HINT2 mRNA translation [J]. Mol Cancer, 2019, 18(1): 161-161. 
39. Lin S, Choe J, Du P, Triboulet R, Gregory R I. The m(6)A Methyltransferase METTL3 Promotes Translation in Human Cancer Cells [J]. Mol Cell, 2016, 62(3): 335-345.

40. Ma L, Chen T, Zhang X, Miao Y, Tian X, Yu K, Xu X, Niu Y, Guo S, Zhang C, Qiu S, Qiao Y, Fang W, Du L, Yu Y, Wang J. The m(6)A reader YTHDC2 inhibits lung adenocarcinoma tumorigenesis by suppressing SLC7A11dependent antioxidant function [J]. Redox Biol, 2021, 38(101801-101801.

41. Lin W, Wang C, Liu G, Bi C, Wang X, Zhou Q, Jin H. SLC7A11/xCT in cancer: biological functions and therapeutic implications [J]. Am J Cancer Res, 2020, 10(10): 3106-3126.

42. Hu K, Li K, Lv J, Feng J, Chen J, Wu H, Cheng F, Jiang W, Wang J, Pei H, Chiao P J, Cai Z, Chen Y, Liu M, Pang X. Suppression of the SLC7A11/glutathione axis causes synthetic lethality in KRAS-mutant lung adenocarcinoma [J]. J Clin Invest, 2020, 130(4): 1752-1766.

43. Lim J K M, Delaidelli A, Minaker S W, Zhang H-F, Colovic M, Yang H, Negri G L, von Karstedt S, Lockwood W W, Schaffer P, Leprivier G, Sorensen P H. Cystine/glutamate antiporter xCT (SLC7A11) facilitates oncogenic RAS transformation by preserving intracellular redox balance [J]. Proc Natl Acad Sci U S A, 2019, 116(19): 9433-9442.

44. Stockwell B R, Friedmann Angeli J P, Bayir H, Bush A I, Conrad M, Dixon S J, Fulda S, Gascón S, Hatzios S K, Kagan V E, Noel K, Jiang X, Linkermann A, Murphy M E, Overholtzer M, Oyagi A, Pagnussat G C, Park J, Ran Q, Rosenfeld 

Biology, and Disease [J]. Cell, 2017, 171(2): 273-285.

45. Miotto G, Rossetto M, Di Paolo M L, Orian L, Venerando R, Roveri A, Vučković A-M, Bosello Travain V, Zaccarin M, Zennaro L, Maiorino M, Toppo S, Ursini F, Cozza G. Insight into the mechanism of ferroptosis inhibition by ferrostatin-1 [J]. Redox Biol, 2020, 28(101328-101328.

46. Zhao $Y$, Shi $Y$, Shen $H$, Xie W. m(6)A-binding proteins: the emerging crucial performers in epigenetics [J]. J Hematol Oncol, 2020, 13(1): 35-35.

47. Lin X, Chai G, Wu Y, Li J, Chen F, Liu J, Luo G, Tauler J, Du J, Lin S, He C, Wang H. RNA m(6)A methylation regulates the epithelial mesenchymal transition of cancer cells and translation of Snail [J]. Nat Commun, 2019, 10(1): 2065-2065.

\section{Figure legends}

Figure 1 METTL3 regulates $\mathrm{m}^{6} \mathrm{~A}$ level in LUAD patients and NSCLC cells.

(A) Representative IHC images and IHC scores of $\mathrm{m}^{6} \mathrm{~A}$ level in 21 LUAD tissues and adjacent normal tissues (Scale bar: $50 \mu \mathrm{m}$ ). (B) Expression of METTL3 in LUAD patients based on TCGA samples using UALCAN platform. (C) Expression of METTL3 in LUAD patients based on GEO datasets (GSE2514). 
and adjacent normal tissues (Scale bar: $50 \mu \mathrm{m})$. (E and F) Relative mRNA levels measured by RT-qPCR and protein levels analyzed by western blot of METTL3

751 in NSCLC cell lines (H1975, A549, PC9, H322, H460, SPC-A1 and H1299),

752 compared with normal human lung epithelia cells (BEAS-2B). (G) Quantitative 753 analysis of the percentage of $\mathrm{m}^{6} \mathrm{~A}$ content in LUAD cells (H1975 and PC9), 754 compared with BEAS-2B cells. $(\mathrm{H})$ Quantitative analysis of the percentage of $755 \mathrm{~m}^{6} \mathrm{~A}$ content in METTL3 stable knockdown H1975 and PC9 cells. (I) 756 Quantitative analysis of the percentage of $\mathrm{m}^{6} \mathrm{~A}$ content in METTL3 757 overexpression $\mathrm{H} 322$ cells. (J) Transfection efficiencies of METTL3 stable 758 knockdown in H1975 and PC9 cells were confirmed by western blot. (K) The 759 overexpression efficiency of METTL3 after $24 \mathrm{~h}$ transfection of METTL3 or vector plasmid in H322 cells. ${ }^{*} \mathrm{P}<0.05$, ${ }^{* *} \mathrm{P}<0.01$, ${ }^{* *} \mathrm{P}<0.001$, ns, not significant.

Figure 2 METTL3 promotes LUAD proliferation and inhibits cell apoptosis.

763 (A) The knockdown efficiency of METTL3 after $48 \mathrm{~h}$ transfection of METTL3 siRNA (si\#1, si\#2) and negative control siRNA (siNC) in H1975 and PC9 cells were confirmed by western blot. (B and $C$ ) CCK- 8 assays showed the growth curves of LUAD cells upon METTL3 knockdown in H1975 and PC9 cells, and METTL3 overexpression in H322 cells respectively. (D and E) Representative images of EdU staining and the ratio of EdU positive cells to total Hoechest 33342 positive cells (Scale bar: $100 \mu \mathrm{m})$. (F and G) Flow cytometry analysis for cell cycle of cellular distribution by PI staining with METTL3 knockdown in 
H1975 and PC9 cells, and METTL3 overexpression in H322 cells. (H and I) Flow cytometry analysis for apoptotic cell proportion (Q2+Q3) by Annexin VFITC/PI staining. (J) Photograph of dissected subcutaneous tumors at sacrificed time. (K) The tumor growth curves of subcutaneous xenograft models with stable METTL3 knockdown (shMETTL3) and negative control (shNC) PC9 cells injection $(n=6)$. (L) Tumor weight of dissected subcutaneous tumors at sacrificed time. (M) Representative IHC images and IHC scores of METTL3 and Ki67 stained in xenograft tumors (Scale bar: $50 \mu \mathrm{m}$ ). (N) Representative TUNEL

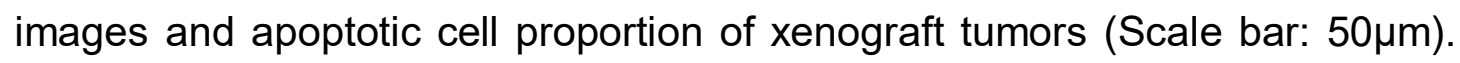
${ }^{* *} \mathrm{P}<0.01,{ }^{* *} \mathrm{P}<0.001$

Figure 3 SLC7A11 acts as a target of METTL3 depending on its $m^{6} A$ methyltransferase activity.

(A) GO analysis of MeRIP-seq data. (B and C) Relative mRNA levels measured by RT-qPCR of selected genes in METTL3 knockdown H1975 and PC9 cells. (D) The relative abundance of $\mathrm{m}^{6} \mathrm{~A}$ peaks of SLC7A11 mRNA in METTL3 knockdown and control PC9 cells analyzed by MeRIP-seq. (E) The protein levels showed by western blot of SLC7A11 with METTL3 knockdown in H1975 and PC9 cells. (F and G) The expression level of SLC7A11 mRNA and protein showed by RT-qPCR and western blot in METTL3 overexpression H322 cells. (H) Representative IHC images and IHC scores of SLC7A11 stained in xenograft tumors (Scale bar: $50 \mu \mathrm{m}$ ). (I and J) The $\mathrm{m}^{6} \mathrm{~A}$ levels of SLC7A11 mRNA measured by MeRIP-qPCR analysis after METTL3 knockdown or 
METTL3 overexpression. ( $\mathrm{K}$ and $\mathrm{L}$ ) SLC7A11 mRNA half-lives $\left(\mathrm{t}_{1 / 2}\right)$ showed by RNA decay rates followed by RT-qPCR after METTL3 knockdown in H1975 and PC9 cells and METTL3 overexpression in H322 cells. Data were collected at indicated timepoints $(0 \mathrm{~h}, 3 \mathrm{~h}$, and $6 \mathrm{~h}$ ) with actinomycin $D($ Act $D, 5 \mu \mathrm{g} / \mathrm{mL}$ ) treatment. ${ }^{* *} \mathrm{P}<0.01,{ }^{* * *} \mathrm{P}<0.001$

Figure 4 METTL3 inhibits LUAD ferroptosis through SLC7A11 $\mathrm{m}^{6} \mathrm{~A}$ modification.

(A) KEGG pathway enrichment analysis of MeRIP-seq data. (B and C) Flow cytometry analysis of intracellular ROS levels measured by DFCH-DA fluorescence with transfection of METTL3 siRNA or NC siRNA in H1975 and PC9 cells (48 h), and METTL3 overexpression in H322 cells (24 h). (D and E) Intracellular MDA levels normalized to corresponding protein contents in METTL3 knockdown or overexpression cells. (F-H) Intracellular ROS and MDA levels in METTL3 stable knockdown H1975 and PC9 cells with SLC7A11 overexpression or Fer-1 treatment $(1 \mu \mathrm{M})$ for $48 \mathrm{~h}$. (I) The transfection efficiency of SLC7A11 overexpression in METTL3 stable knockdown H1975 and PC9 cells was confirmed by western blot. (J) CCK-8 assays showed the growth curves of H1975 and PC9 cells for 3 days after indicated treatments (Fer-1, 1 $\mu \mathrm{M})$. (K) Representative images of EdU staining and the ratio of EdU positive cells to total Hoechest 33342 positive cells in METTL3 stable knockdown PC9 cells after SLC7A11 overexpression or Fer-1 treatment $(1 \mu \mathrm{M})$ for $48 \mathrm{~h}$,

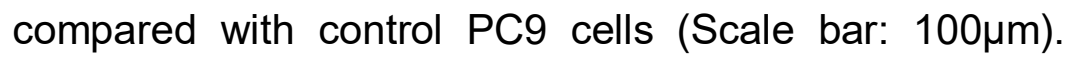

(L) Flow cytometry 
analysis for apoptotic cell proportion (Q2+Q3) by Annexin V-FITC/PI staining. ${ }^{*} \mathrm{P}<0.05,{ }^{* *} \mathrm{P}<0.01,{ }^{* *} \mathrm{P}<0.001$.

Figure 5 YTHDF1 is recruited by METTL3 to enhance SLC7A11 mRNA stability and translation.

( $A$ and $B$ ) The knockdown efficiency after $48 \mathrm{~h}$ transfection of YTHDF1 siRNA and negative control siRNA (siNC) in PC9 cells was confirmed by RT-qPCR and western blot. ( $C$ and $D$ ) The relative mRNA expression determined by RT-qPCR and the protein expression showed by western blot of SLC7A11 after YTHDF1 knockdown in PC9 cells. (E) SLC7A11 mRNA half-lives ( $\left.\mathrm{t}_{1 / 2}\right)$ showed by RNA decay rates followed by RT-qPCR after YTHDF1 knockdown in PC9 cells. Data were collected at indicated timepoints $(0 \mathrm{~h}, 3 \mathrm{~h}$, and $6 \mathrm{~h})$ with actinomycin $\mathrm{D}$ (Act $\mathrm{D}, 5 \mu \mathrm{g} / \mathrm{mL}$ ) treatment. (F) RIP-qPCR revealed the binding enrichment of YTHDF1 to SLC7A11 in METTL3 stable knockdown and negative control PC9 cells. (G) The protein levels of SLC7A11 showed by western blot in YTHDF1 knockdown H322 cells with METTL3 overexpression, compared with control H322 cells. (H) Flow cytometry analysis of intracellular ROS levels in YTHDF1 knockdown H322 cells with METTL3 overexpression, compared with control H322 cells. (I) Flow cytometry analysis for apoptotic cell proportion (Q2+Q3) by Annexin V-FITC/PI staining in $\mathrm{H} 322$ cells. ${ }^{* *} \mathrm{P}<0.01$, ${ }^{* *} \mathrm{P}<0.001$, ns, not significant.

Figure 6 METTL3-mediated $\mathrm{m}^{6} \mathrm{~A}$ modification of SLC7A11 is related to LUAD clinically. 
837 (A and B) Representative IHC images and IHC scores of YTHDF1 and

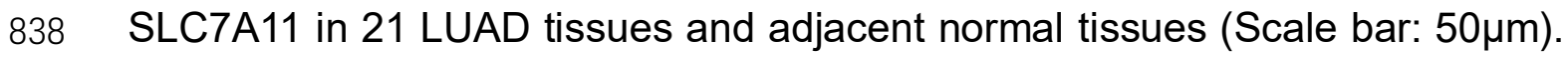
839 (C and D) Expression of YTHDF1 and SLC7A11 in LUAD patients based on 840 TCGA samples using UALCAN platform. (E) The percentages of specimens 841 with high or low METTL3 expression relative to the levels of YTHDF1 and 842 SLC7A11. (F) The percentages of specimens with high or low $\mathrm{m}^{6} \mathrm{~A}$ expression 843 relative to the levels of YTHDF1 and SLC7A11. (G) Kaplan-Meier OS analysis 844 of SLC7A11 expression in LUAD patients. (http://kmplot.com/analysis/)

Supplementary Materials

847 Figure S1 (A-D) Expression of METTL14, WTAP, ZC3H13 and RBM15 in LUAD

848 patients based on TCGA samples using UALCAN platform.

849 Table S1 The sequence of primers for qPCR 


\section{Figures}

(A)

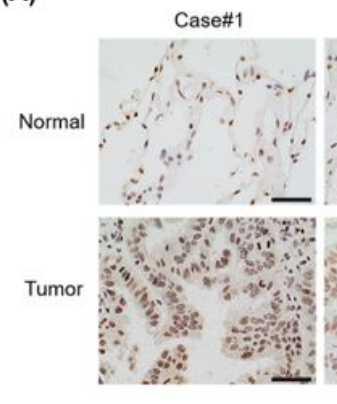

(C)

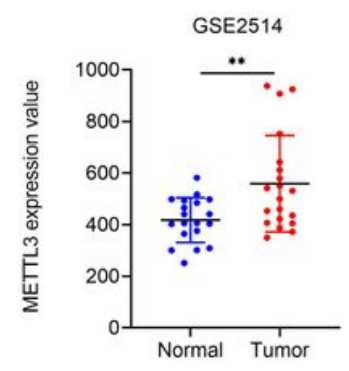

(E)

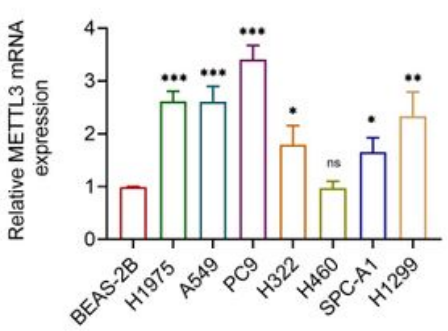

(H)

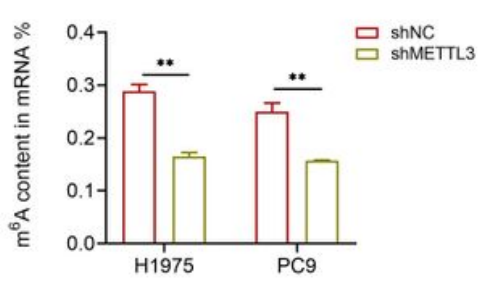

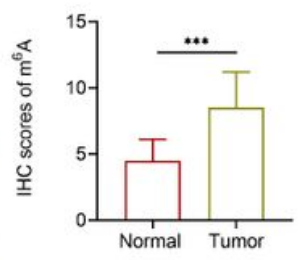

(B) Expression of METTL3 in LUAD based on sample types

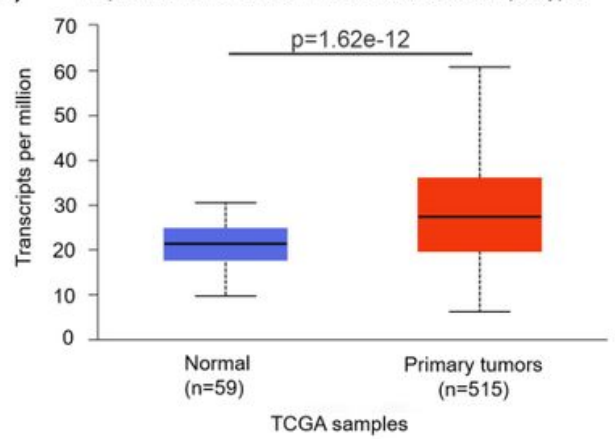

(D)
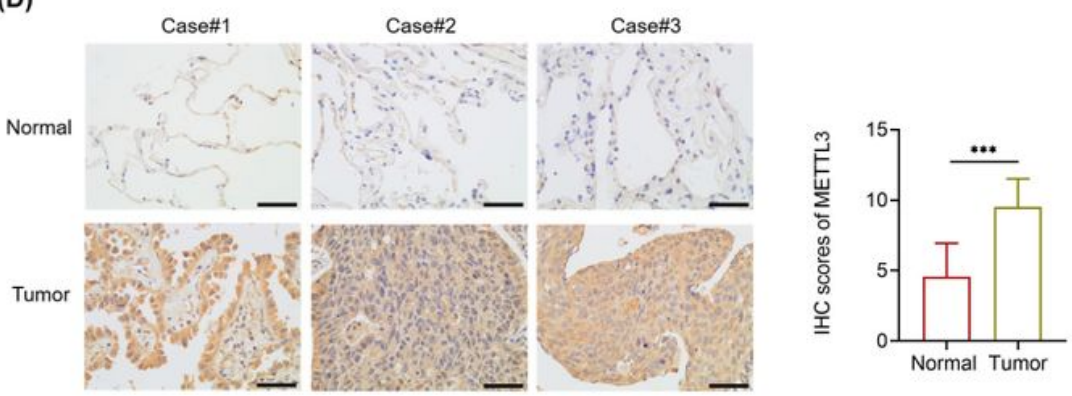

(F)

(G)
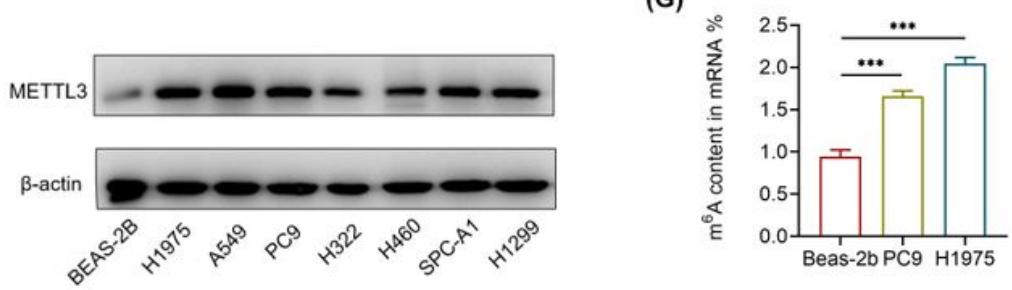

(I)

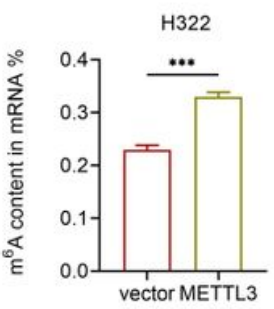

(J)

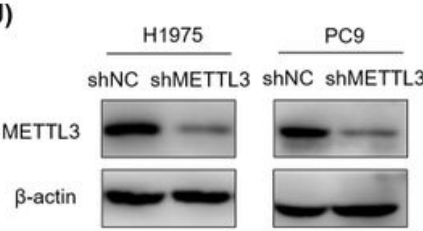

(K)

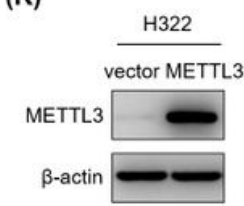

\section{Figure 1}

METTL3 regulates m6 A level in LUAD patients and NSCLC cells. (A) Representative IHC images and IHC scores of m6 A level in 21 LUAD tissues and adjacent normal tissues (Scale bar: $50 \mu m)$. (B) Expression of METTL3 in LUAD patients based on TCGA samples using UALCAN platform. (C) Expression of METTL3 in LUAD patients based on GEO datasets (GSE2514). (D) Representative IHC images and IHC scores of

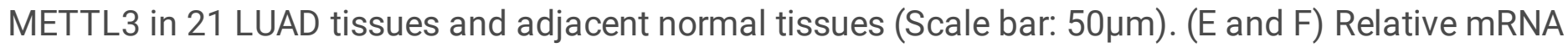
levels measured by RT-qPCR and protein levels analyzed by western blot of METTL3 in NSCLC cell lines (H1975, A549, PC9, H322, H460, SPC-A1 and H1299), compared with normal human lung epithelia cells (BEAS-2B). (G) Quantitative analysis of the percentage of m6 A content in LUAD cells (H1975 and PC9), 
compared with BEAS-2B cells. $(\mathrm{H})$ Quantitative analysis of the percentage of m6 A content in METTL3 stable knockdown $\mathrm{H} 1975$ and PC9 cells. (I) Quantitative analysis of the percentage of m6 A content in METTL3 overexpression H322 cells. (J) Transfection efficiencies of METTL3 stable knockdown in H1975 and PC9 cells were confirmed by western blot. (K) The overexpression efficiency of METTL3 after $24 \mathrm{~h}$ transfection of METTL3 or vector plasmid in H322 cells. ${ }^{*} P<0.05$, ${ }^{*} P<0.01,{ }^{*} * \mathrm{P}<0.001$, ns, not significant.

(A)

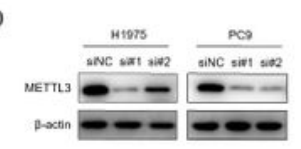

(C)

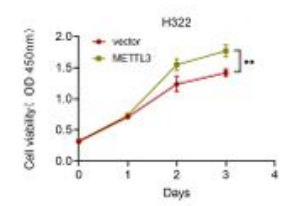

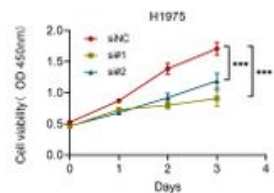

(D)

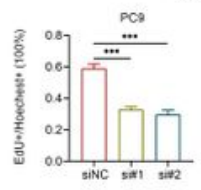

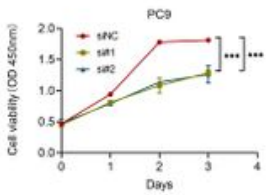
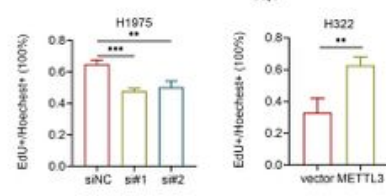

(E)
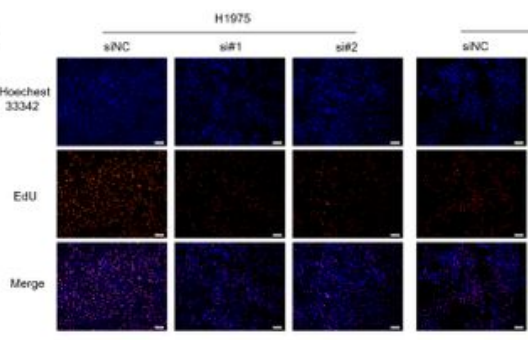

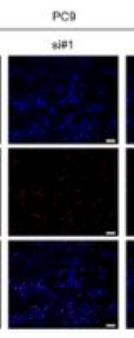

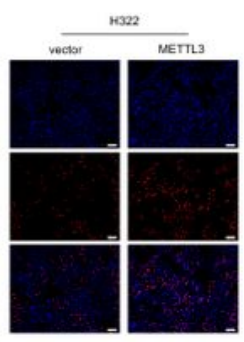

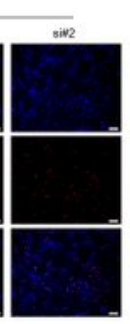

(G)
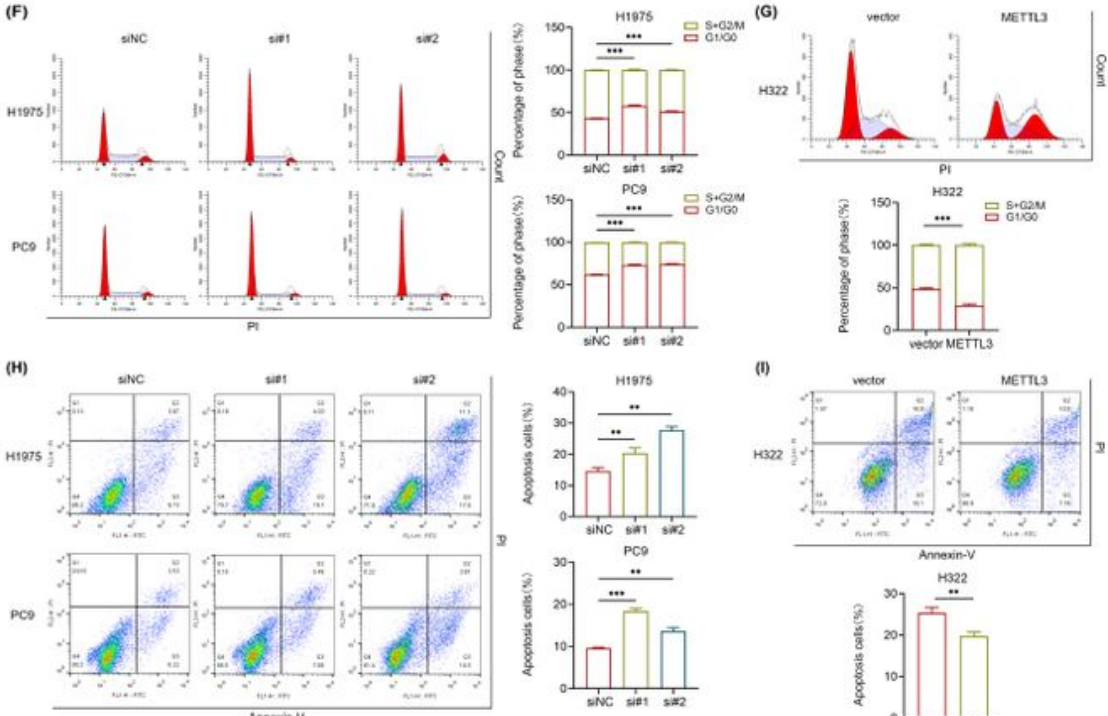

(I)
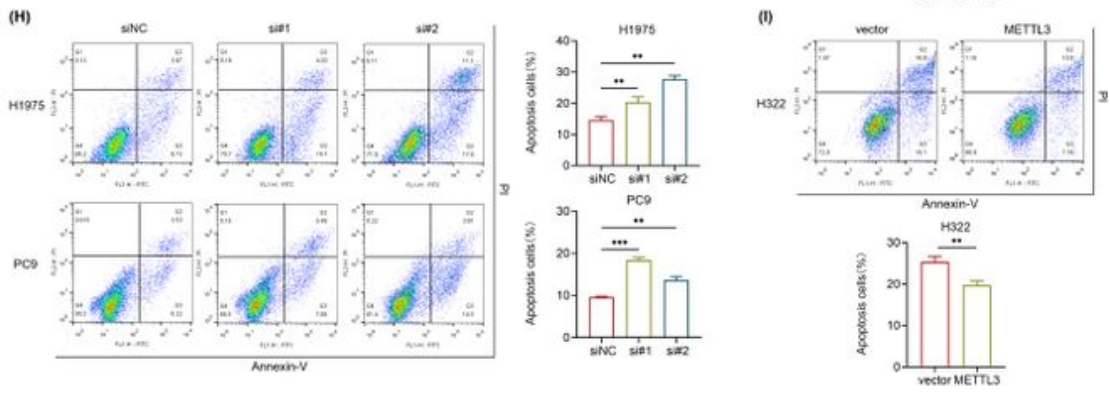

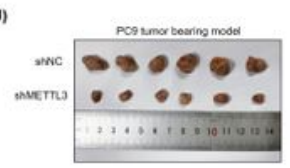

(M)

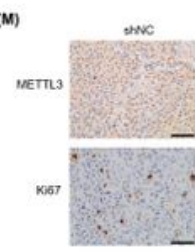

(K)

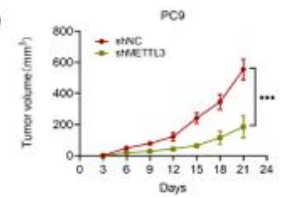

(L)
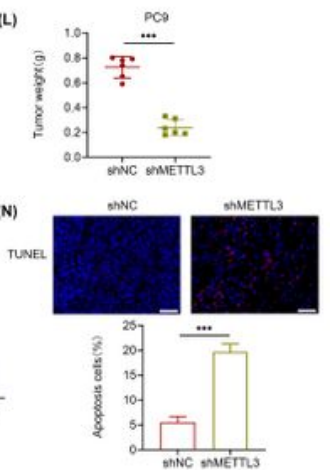

Figure 2 
METTL3 promotes LUAD proliferation and inhibits cell apoptosis. (A) The knockdown efficiency of METTL3 after $48 \mathrm{~h}$ transfection of METTL3 siRNA (si\#1, si\#2) and negative control siRNA (siNC) in H1975 and PC9 cells were confirmed by western blot. (B and C) CCK-8 assays showed the growth curves of LUAD cells upon METTL3 knockdown in H1975 and PC9 cells, and METTL3 overexpression in H322 cells respectively. ( $D$ and $E$ ) Representative images of EdU staining and the ratio of EdU positive cells to total Hoechest 33342 positive cells (Scale bar: $100 \mu \mathrm{m}$ ). ( $\mathrm{F}$ and G) Flow cytometry analysis for cell cycle of cellular distribution by PI staining with METTL3 knockdown in H1975 and PC9 cells, and METTL3 overexpression in $\mathrm{H} 322$ cells. ( $\mathrm{H}$ and I) Flow cytometry analysis for apoptotic cell proportion (Q2+Q3) by Annexin VFITC/PI staining. (J) Photograph of dissected subcutaneous tumors at sacrificed time. (K) The tumor growth curves of subcutaneous xenograft models with stable METTL3 knockdown (shMETTL3) and negative control (shNC) PC9 cells injection ( $n=6)$. (L) Tumor weight of dissected subcutaneous tumors at sacrificed time. (M) Representative IHC images and IHC scores of METTL3 and Ki67 stained in xenograft tumors (Scale bar: $50 \mu \mathrm{m}$ ). (N) Representative TUNEL images and apoptotic cell proportion of xenograft tumors (Scale bar: $50 \mu \mathrm{m}) .{ }^{* *} \mathrm{P}<0.01,{ }^{* \star *} \mathrm{P}<0.001$. 
(A)

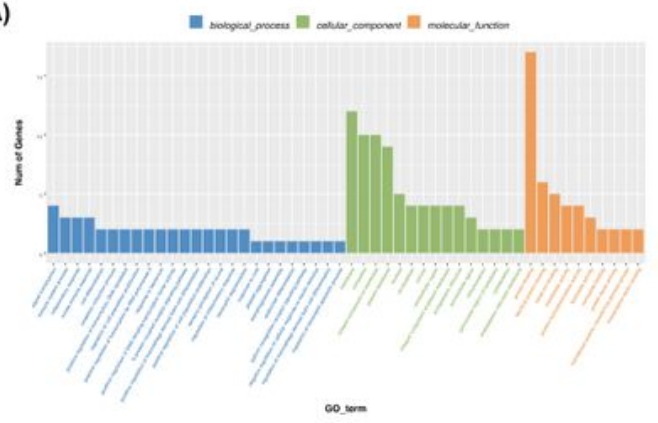

(C)

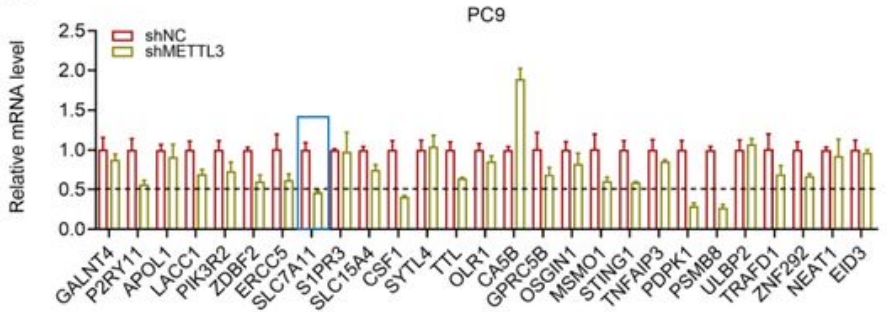

(B)

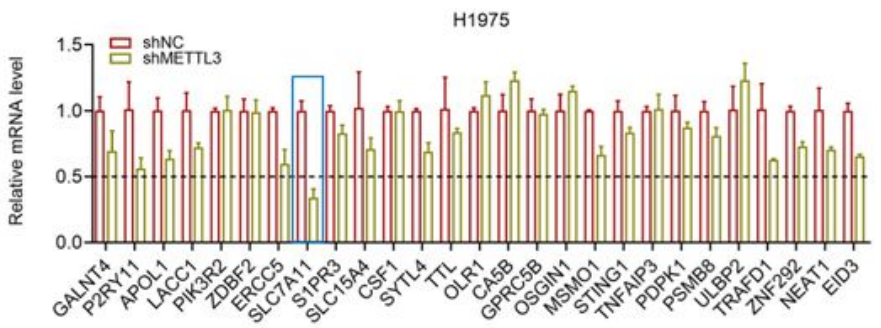

(D)

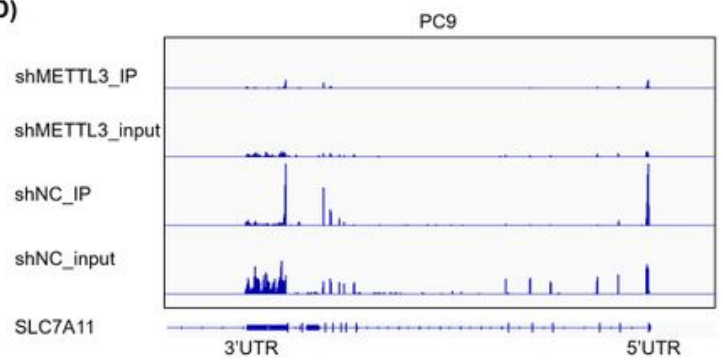

(E)

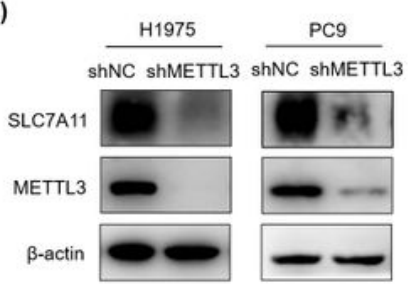

(F)

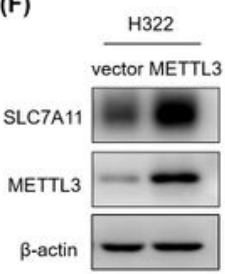

(G)

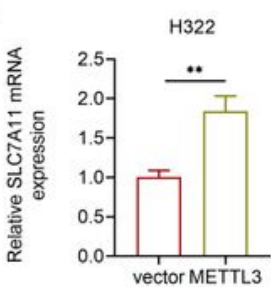

(H)

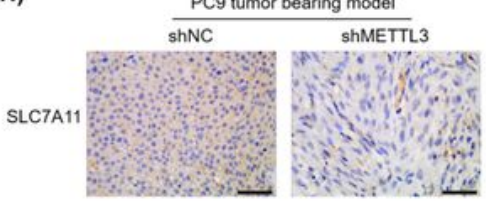

(J)
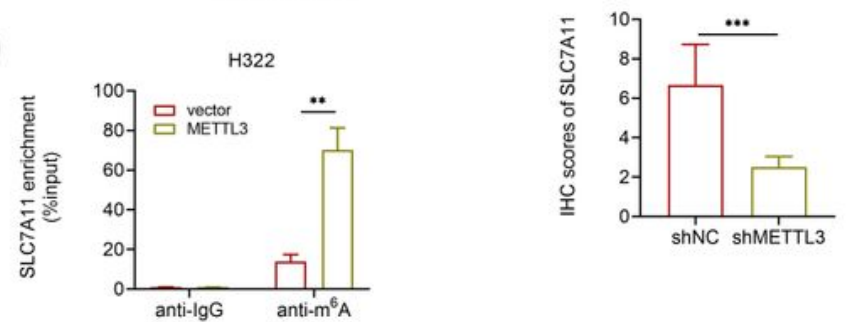

(K)

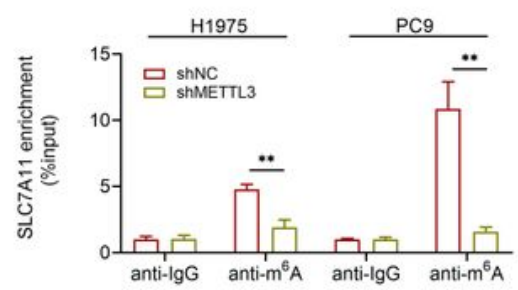

PC9
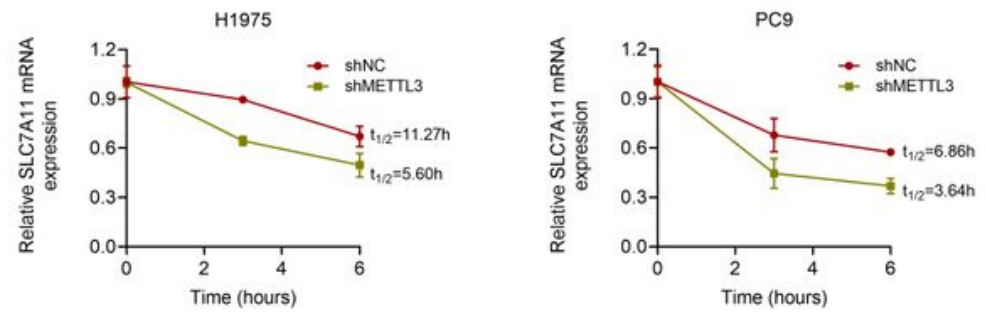

(L)

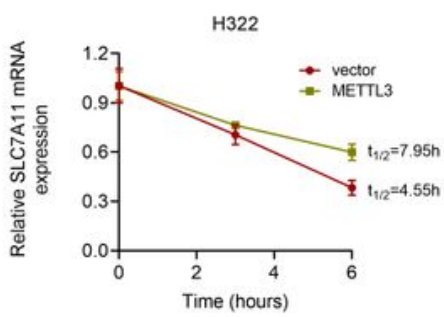

\section{Figure 3}

SLC7A11 acts as a target of METTL3 depending on its m6 A methyltransferase activity. (A) G0 analysis of MeRIP-seq data. (B and C) Relative mRNA levels measured by RT-qPCR of selected genes in METTL3 knockdown $\mathrm{H} 1975$ and PC9 cells. (D) The relative abundance of m6 A peaks of SLC7A11 mRNA in METTL3 knockdown and control PC9 cells analyzed by MeRIP-seq. (E) The protein levels showed by western blot of SLC7A11 with METTL3 knockdown in H1975 and PC9 cells. (F and G) The expression 
level of SLC7A11 mRNA and protein showed by RT-qPCR and western blot in METTL3 overexpression H322 cells. (H) Representative IHC images and IHC scores of SLC7A11 stained in xenograft tumors (Scale bar: $50 \mu \mathrm{m})$. (I and $\mathrm{J}$ ) The m6 A levels of SLC7A11 mRNA measured by MeRIP-qPCR analysis after METTL3 knockdown or METTL3 overexpression. ( $K$ and $L$ ) SLC7A11 mRNA half-lives (t1/2) showed by RNA decay rates followed by RT-qPCR after METTL3 knockdown in $\mathrm{H} 1975$ and PC9 cells and METTL3 overexpression in $\mathrm{H} 322$ cells. Data were collected at indicated timepoints $(0 \mathrm{~h}, 3 \mathrm{~h}$, and $6 \mathrm{~h})$ with actinomycin $D($ Act $D, 5 \mu \mathrm{g} / \mathrm{mL})$ treatment. ${ }^{*} \mathrm{P}<0.01$, ${ }^{* \star *} \mathrm{P}<0.001$.
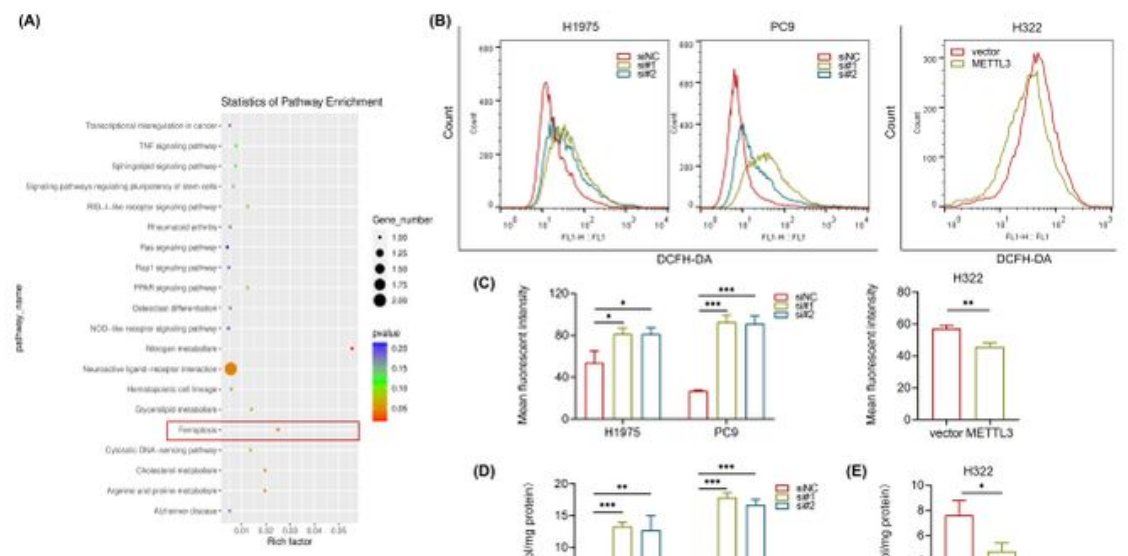

(F)

(D)
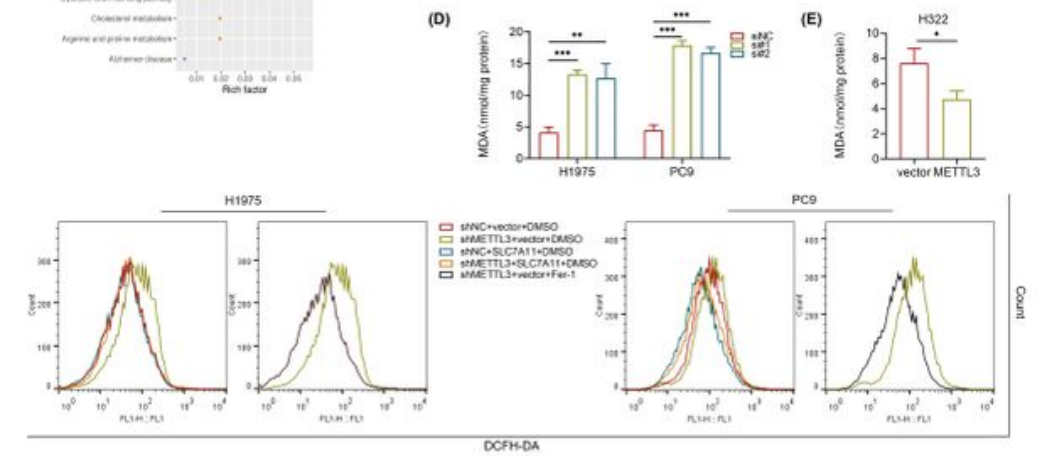

(G)
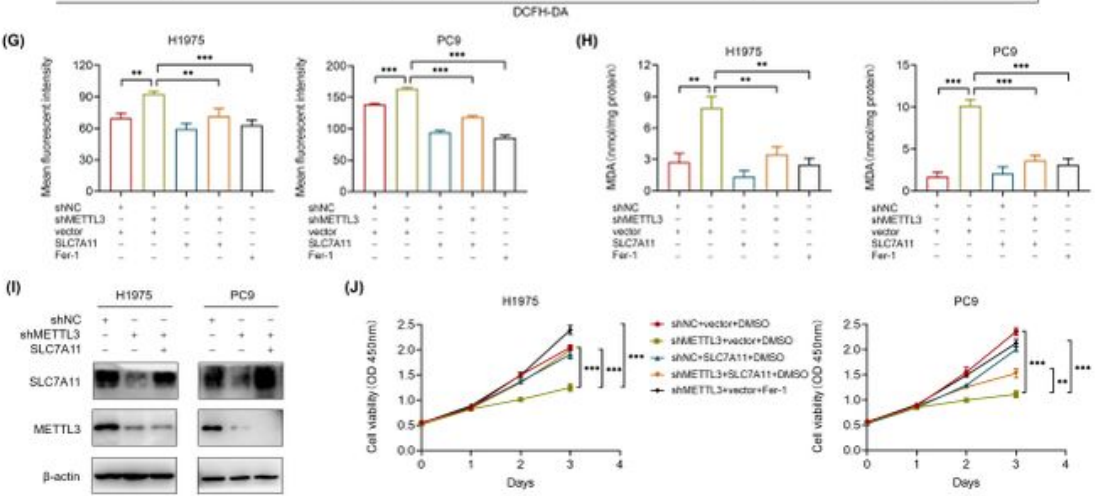

(K)
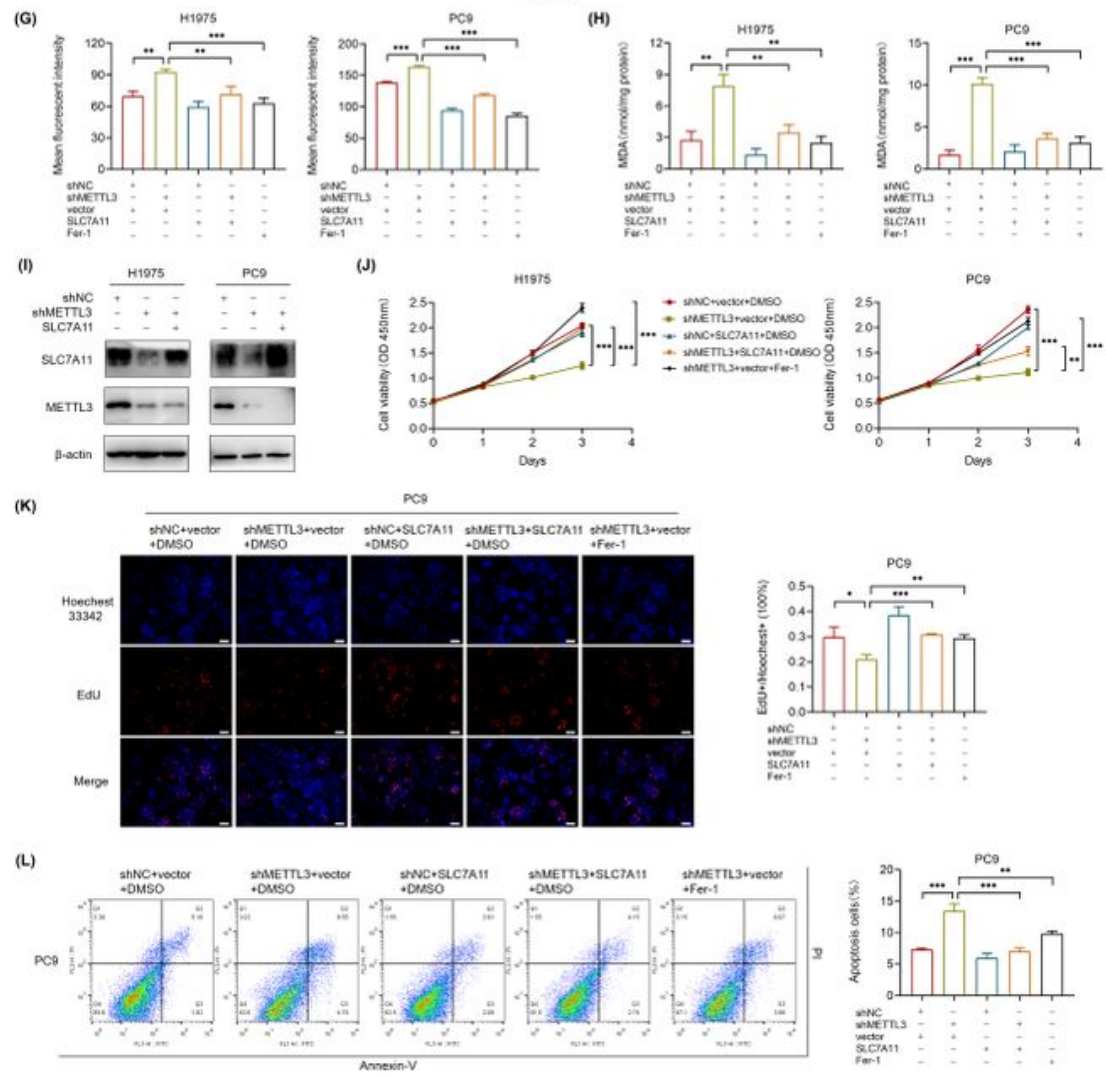


\section{Figure 4}

METTL3 inhibits LUAD ferroptosis through SLC7A11 m6 798 A modification. (A) KEGG pathway enrichment analysis of MeRIP-seq data. (B and C) Flow cytometry analysis of intracellular ROS levels measured by DFCH-DA fluorescence with transfection of METTL3 siRNA or NC siRNA in H1975 and PC9 cells (48 h), and METTL3 overexpression in H322 cells (24 h). (D and E) Intracellular MDA levels normalized to corresponding protein contents in METTL3 knockdown or overexpression cells. (F-H) Intracellular ROS and MDA levels in METTL3 stable knockdown H1975 and PC9 cells with SLC7A11 overexpression or Fer-1 treatment $(1 \mu \mathrm{M})$ for $48 \mathrm{~h}$. (I) The transfection efficiency of SLC7A11 overexpression in METTL3 stable knockdown H1975 and PC9 cells was confirmed by western blot. (J) CCK-8 assays showed the growth curves of $\mathrm{H} 1975$ and PC9 cells for 3 days after indicated treatments (Fer-1, $1 \mu \mathrm{M})$. (K) Representative images of EdU staining and the ratio of EdU positive cells to total Hoechest 33342 positive cells in METTL3 stable knockdown PC9 cells after SLC7A11 overexpression or Fer-1 treatment $(1 \mu \mathrm{M})$ for $48 \mathrm{~h}$, compared with control PC9 cells (Scale bar: $100 \mu \mathrm{m})$. (L) Flow cytometry analysis for apoptotic cell proportion (Q2+Q3) by Annexin V-FITC/PI staining. ${ }^{*} \mathrm{P}<0.05,{ }^{\star \star} \mathrm{P}<0.01$, ${ }^{\star \star \star} \mathrm{P}<$ 0.001 . 
(A)

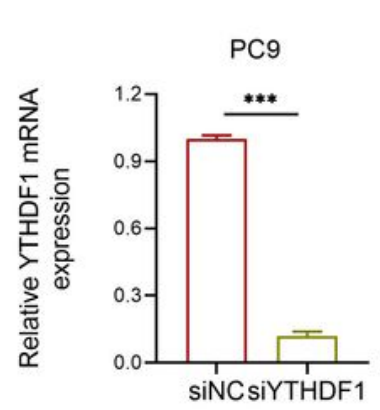

(E)

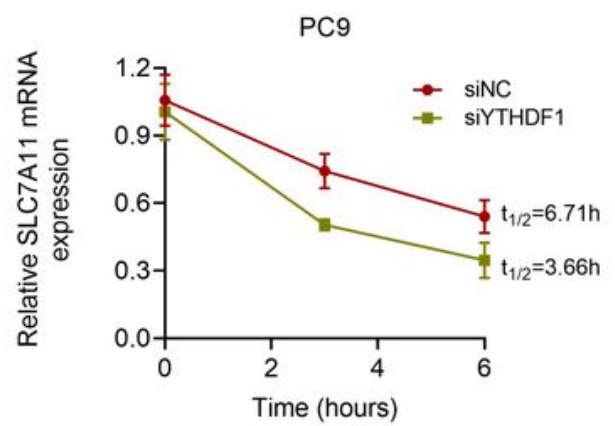

(H)

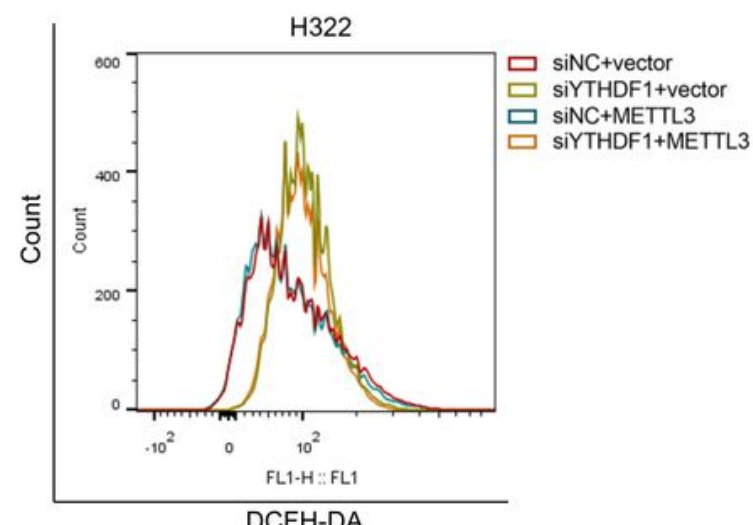

(F)
(C)

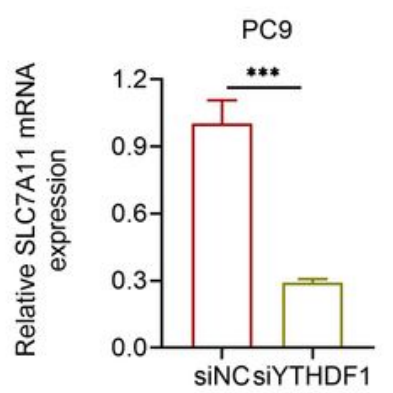

(D)

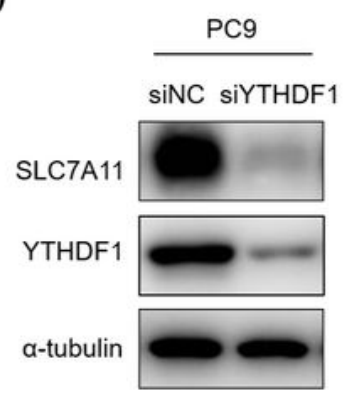

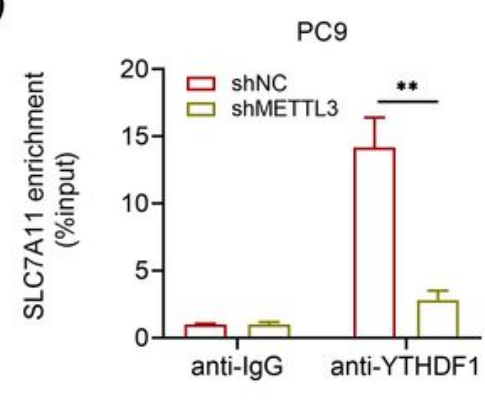

(G)

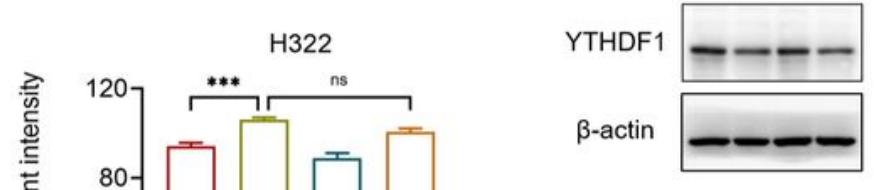

(I)

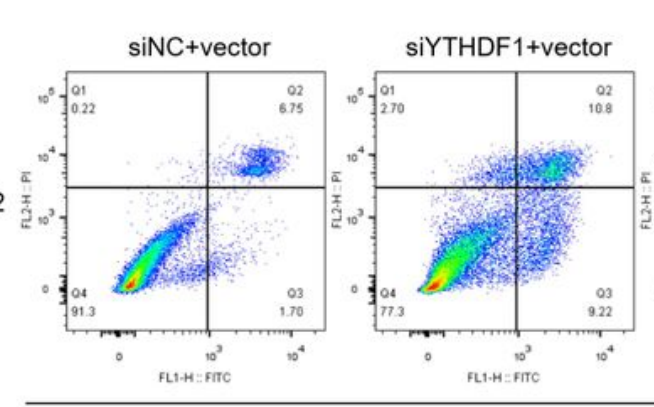

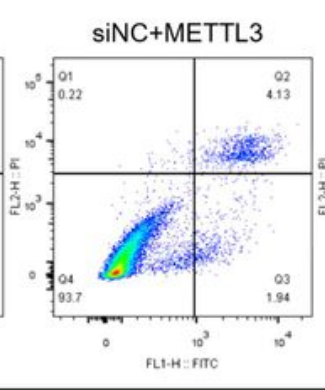

Annexin-V
H322

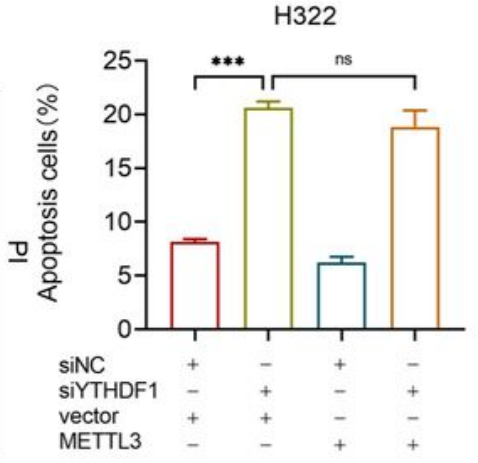

\section{Figure 5}

YTHDF1 is recruited by METTL3 to enhance SLC7A11 mRNA stability and translation. (A and B) The knockdown efficiency after 48h transfection of YTHDF1 siRNA and negative control siRNA (siNC) in PC9 cells was confirmed by RT-qPCR and western blot. ( $C$ and $D)$ The relative mRNA expression determined by RT-qPCR and the protein expression showed by western blot of SLC7A11 after YTHDF1 knockdown in PC9 cells. (E) SLC7A11 mRNA half-lives (t1/2) showed by RNA decay rates followed by RT-qPCR after 
YTHDF1 knockdown in PC9 cells. Data were collected at indicated timepoints $(0 \mathrm{~h}, 3 \mathrm{~h}$, and $6 \mathrm{~h})$ with actinomycin D (Act D, $5 \mu \mathrm{g} / \mathrm{mL}$ ) treatment. (F) RIP-qPCR revealed the binding enrichment of YTHDF1 to SLC7A11 in METTL3 stable knockdown and negative control PC9 cells. (G) The protein levels of SLC7A11 showed by western blot in YTHDF1 knockdown H322 cells with METTL3 overexpression, compared with control H322 cells. (H) Flow cytometry analysis of intracellular ROS levels in YTHDF1 knockdown H322 cells with METTL3 overexpression, compared with control H322 cells. (I) Flow cytometry analysis for apoptotic cell proportion (Q2+Q3) by Annexin V-FITC/PI staining in H322 cells. ${ }^{\star \star} \mathrm{P}<0.01,{ }^{\star \star \star} \mathrm{P}<0.001$, ns, not significant.
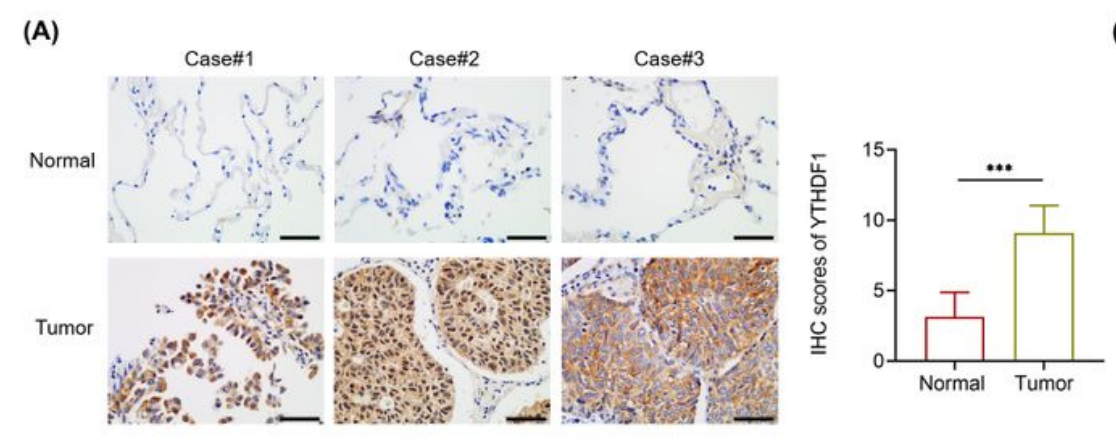

(C)
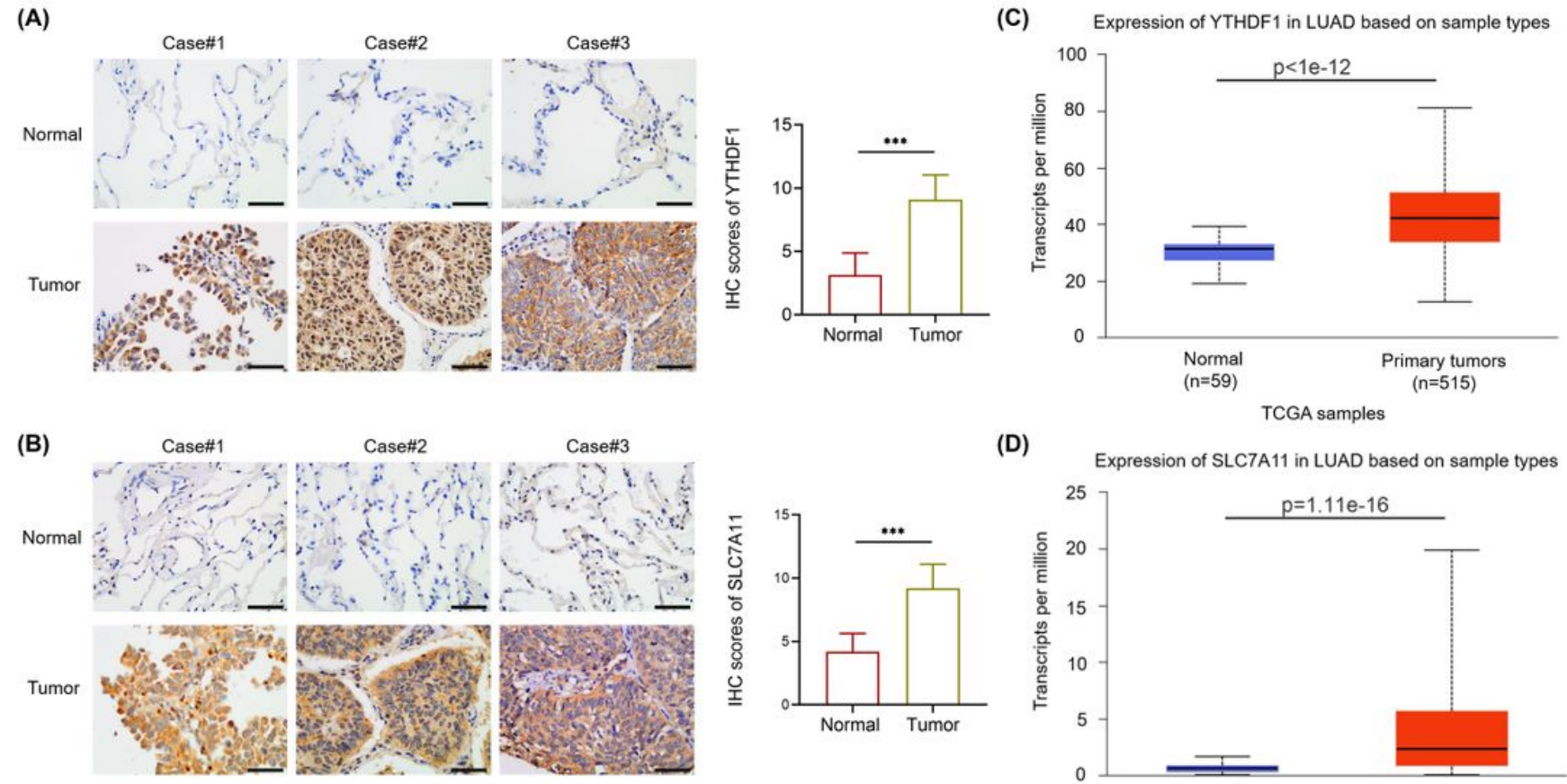

(D)

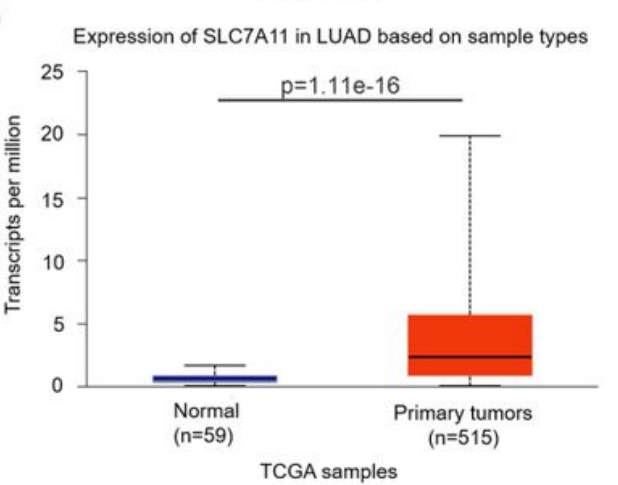

(E)
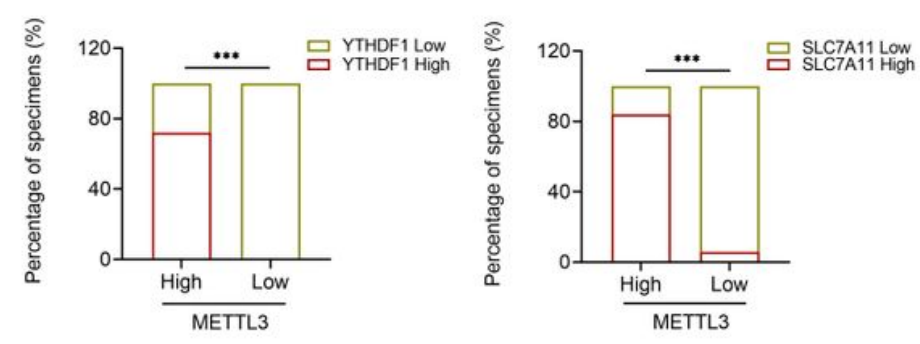

(F)
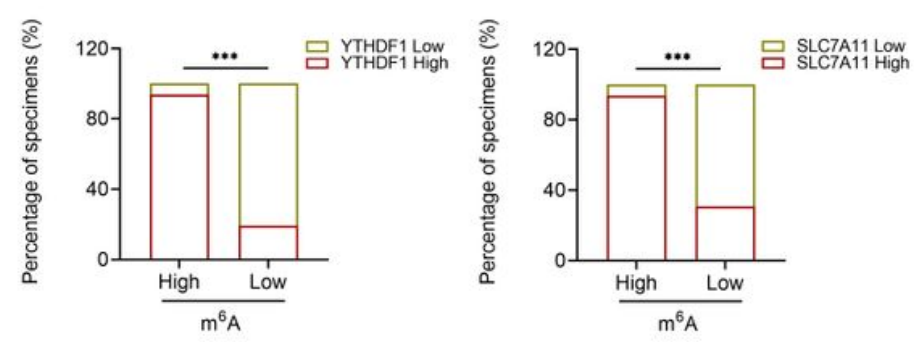

(G)

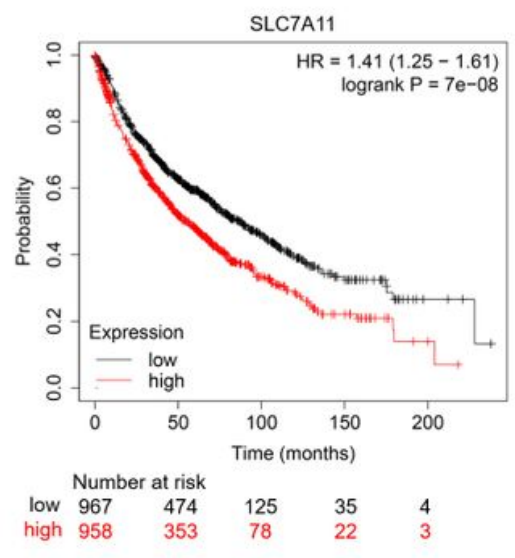

Figure 6

METTL3-mediated m6 835 A modification of SLC7A11 is related to LUAD clinically. (A and B) Representative IHC images and IHC scores of YTHDF1 and SLC7A11 in 21 LUAD tissues and adjacent 


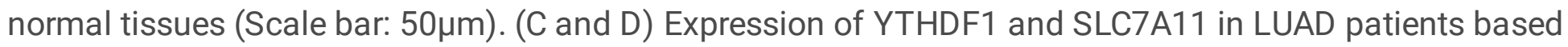
on TCGA samples using UALCAN platform. (E) The percentages of specimens with high or low METTL3 expression relative to the levels of YTHDF1 and SLC7A11. (F) The percentages of specimens with high or low m6 A expression relative to the levels of YTHDF1 and SLC7A11. (G) Kaplan-Meier OS analysis of SLC7A11 expression in LUAD patients. (http://kmplot.com/analysis/)

\section{Supplementary Files}

This is a list of supplementary files associated with this preprint. Click to download.

- Figures1.rar

- supplementarytables1.pdf 